\title{
Incremental stability of hybrid dynamical systems
}

\author{
J. J. Benjamin Biemond, Romain Postoyan, W. P. Maurice H. Heemels, and Nathan van de Wouw
}

\begin{abstract}
The analysis of incremental stability typically involves measuring the distance between any two solutions of a given dynamical system at the same time instant, which is problematic when studying hybrid dynamical systems. Indeed, hybrid systems generate solutions defined with respect to hybrid time instances (that consists of both the continuous time elapsed and the discrete time, which is the number of jumps experienced so far), and two solutions of the same hybrid system may not be defined at the same hybrid time instant. To overcome this issue, we present novel definitions of incremental stability for hybrid systems based on graphical closeness of solutions. As we will show, defining incremental asymptotic stability with respect to the hybrid time yields a restrictive notion, such that we also investigate incremental asymptotic stability notions with respect to the continuous time only or the discrete time only, respectively. In this manner, two (effectively dual) incremental stability notions are attained, called jump- and flow incremental asymptotic stability. To present Lyapunov conditions for these two notions, in both cases, we resort to an extended hybrid system and we prove that the stability of a well-defined set for this extended system implies incremental stability of the original system. We can then use available Lyapunov conditions to infer the set stability of the extended system. Various examples are provided throughout the paper, including an event-triggered control application and a bouncing ball system with Zeno behaviour, that illustrate incremental stability with respect to continuous time or discrete time, respectively.
\end{abstract}

Index Terms-Incremental stability, Hybrid systems, Lyapunov stability.

\section{INTRODUCTION}

A dynamical system is said to be incrementally asymptotically stable when all its solutions are asymptotically stable, see e.g., [1]-[4]. Loosely speaking, this means that (i) the states of any two solutions, whose initial conditions are 'close' to each other, remain 'close' to each other for all positive times and (ii) the states of any two solutions converge towards each other as time proceeds. Incremental stability (and the related notions of convergence [5] and contraction [6]) is a

J.J.B. Biemond is with Department of Optomechatronics, Netherlands Organization for Applied Scientific Research, TNO, Delft, The Netherlands. This research has been performed while he was with the Department of Computer Science, KU Leuven, Belgium. e-mail: benjamin.biemond@kuleuven.be. His work is supported by the FWO Pegasus Marie Curie Fellowship, from FWO G071711N and the Optimization in Engineering Center (OPTEC) of KU Leuven.

R. Postoyan is with the Université de Lorraine, CRAN, UMR 7039 and the CNRS, CRAN, UMR 7039, France. e-mail: romain.postoyan@univlorraine.fr. His work was partially supported by the ANR under the grant SEPICOT (ANR 12 JS03 004 01).

W.P.M.H. Heemels is with the Department of Mechanical Engineering, Eindhoven University of Technology, The Netherlands. e-mail: w.p.m.h.heemels@tue.nl.

N. van de Wouw is with the Department of Mechanical Engineering, Eindhoven University of Technology, The Netherlands, the Department of Civil, Environmental \& Geo-Engineering, University of Minnesota, U.S.A. and the Delft Center for Systems and Control, Delft University of Technology, The Netherlands. e-mail: n.v.d.wouw@tue.nl. key concept that arises in a wide variety of control problems. Examples include synchronisation [7], tracking control and observer design [8], output regulation [5], robustness analysis [9], control reconfiguration of systems with actuator and sensor faults [10], frequency-domain analysis of nonlinear systems [11], model reduction [12], construction of symbolic models for nonlinear control systems [13] and many more.

The majority of the literature on incremental stability (and related stability notions) focuses on smooth continuous-time or discrete-time systems, while some works addressing such stability properties for classes of non-smooth systems can be found in [8], [11], [13]-[15]. The objective of this paper is to investigate incremental stability for hybrid systems, for which results in the literature are rare. Exceptions are the recent works in [16]-[19], where incremental stability is studied for a class of hybrid systems in the formalism of [20]. Results on convergence for a class of measure differential inclusions can be found in [21], [22].

Since the solutions to a hybrid system experience both continuous-time evolution and discrete events, these solutions can be defined on a domain of hybrid time instants, that consists of a pair containing the continuous time elapsed and the number of discrete events [20]. The analysis of incremental stability for hybrid systems in this formalism is challenging for two reasons, both associated with the hybrid nature of the dynamics. Firstly, solutions for the same hybrid system do not necessarily have identical hybrid time domains and, therefore, it is not a priori clear at which hybrid time instants solutions should be compared. Secondly, earlier works in [23], [24] on tracking control problems have shown that, if close solutions may exhibit jumps with a small time mismatch, as generally occurs for hybrid systems with state-triggered jumps, then the Euclidean distance function is not suitable due to the 'peaks' in this distance function.

In this paper, we address both challenges and we first propose an incremental stability definition, which says that the graphs of two maximal solutions with 'close' initial conditions remain 'close' for all hybrid times and converge to each other when hybrid time progresses. To decide when solutions should be considered 'close' as stated in the previous sentence, we exploit the concept of $\varepsilon$-closeness of hybrid arcs (see [20]) and we use a generic mapping to evaluate the distance between the states of the solutions, and not necessarily the Euclidean distance. Consistent with incremental stability definitions for continuous-time in [1] and discrete-time systems in [25], we define incremental stability as a uniform asymptotic stability property.

When the asymptotic behaviour of incremental stability is defined with respect to the hybrid time, a restrictive system property follows as we formally show that then the system is either purely continuous, meaning that all its solutions 
exclusively flow and do not undergo jumps, or it behaves like a discrete-time systems and its solutions exclusively jump. Motivated by this observation, we present alternative definitions that relax the requirements on the hybrid time domains of the solutions. We do this by introducing two weaker notions for (pre-)incremental stability, being flow (pre-)incremental asymptotic stability and jump (pre-)incremental asymptotic stability, where the systems satisfy incremental asymptotic stability properties with respect to the continuous time or discrete time, respectively. The analysis of flow incremental stability consists of evaluating the distance between two solutions at 'close' continuous times, while tolerating an offset between the discrete times at which the two solutions are compared. Consequently, flow incremental stability is important for hybrid systems in which the continuous time is more dominant than the discrete time as, e.g., in models of mechanical systems with impacts. In contrast, the definition of jump incremental stability allows an offset between the continuous times while keeping the discrete time instances close. This definition is relevant for systems for which the discrete time is dominant.

We provide sufficient conditions for flow incremental asymptotic stability using the stability analysis of a set for an extended system, as also employed in [1] for continuous-time systems. Solutions of this extended system represent a pair of solutions to the original system, where, by construction, the continuous time elapsed for both solutions are synchronised. The stability of a well-defined set for this extended system is shown to be equivalent to incremental stability with respect to continuous time, provided that the distance between two solutions can always be evaluated at identical continuous times. This may not be feasible when the 'peaking phenomenon' of the distance evaluated at identical continuous times necessitates the comparison of solutions with a small time mismatch. In that case we show, for a class of hybrid systems, that the analysis of flow incremental stability with respect to a specifically constructed distance function, for which no continuous-time mismatch is required, allows to prove flow incremental stability also in the original ('peaking') distance. Exploiting available set-stability analysis techniques, we then provide Lyapunov-based sufficient conditions for flow incremental stability. These results are illustrated with two examples, including an event-triggered control system [26].

Additionally, sufficient conditions for the symmetric notion of jump incremental asymptotic stability are also provided using a different extended hybrid system that synchronises the number of jumps of two solutions. We show that jump incremental asymptotic stability is equivalent to uniform global asymptotic stability of a well-defined set for this extended system, and exploit this equivalence to provide Lyapunov-based sufficient conditions for incremental stability using existing set-stability analysis techniques. These results are applied to the bouncing-ball system to show that the accumulation of jumps (Zeno behaviour) induces jump incremental asymptotic stability.

Finally, the relations between the three definitions are investigated in detail. Moreover, we show that incrementally stable continuous-time and discrete-time systems are flow or jump incrementally stable, respectively, when these systems are embedded in a hybrid system, thereby showing that these notions naturally extend the 'classical' ones.

To relate our work further to the existing literature, note that the notions of incremental asymptotic stability with respect to the hybrid time, as well as jump incremental stability, have not been studied before. However, the incremental stability notion presented in [16], [17], as well as the two alternative notions presented in [18], also prioritise continuous time and, consequently, are related to the concept of flow incremental stability, even though they all differ from flow incremental stability in the following sense. First, in contrast to [18], flow incremental stability does not require to compare two solutions at the same continuous time, but allows a small time mismatch, which is essential to handle the 'peaking' phenomenon. Second, we require a uniform bound on the convergence rate for flow incremental stability, in contrast with [18, Definition 2.2] and [16], [17], which do not provide uniform notions, but is similar to [18, Definition 2.9]. Finally, we allow for a larger class of distance-like functions than in [16]-[18] and allow to consider hybrid systems with non-complete solutions or with solutions that can have two consecutive jumps without flow in between. The characterisation of flow incremental stability in terms of set-stability reminds of the approach in [18], when the assumptions in [18] are satisfied, even though we use a different extended system.

Focusing on systems with complete solutions, in our preliminary work in [19], we have advertised the proposed definitions for incremental stability. In the present paper, we generalise these definitions to systems with non-complete solutions, and, in addition, provide a characterisation of flow and jump incremental stability using extended hybrid systems and setstability results, not given in [19].

The paper is organised as follows. Preliminaries are given in Section II. The definitions of incremental stability in the graphical sense is presented in Section III. We then define flow and jump incremental stability and we provide the associated Lyapunov-based conditions in Sections IV and V, respectively. The relations between the definitions are studied in Section VI and the link with existing notions for continuous-time and discrete-time systems are addressed in Section VII. Section VIII concludes the paper. All the proofs are given in the appendix.

\section{Preliminaries}

Let $\mathbb{R}:=(-\infty, \infty), \quad \mathbb{R}_{\geq 0}:=[0, \infty), \mathbb{Z}:=$ $\{\ldots,-2,-1,0,1,2, \ldots\}, \mathbb{N}_{0}:=\{0,1,2, \ldots\}$ and $\mathbb{N}_{>0}:=$ $\{1,2, \ldots\}$. For $x \in \mathbb{R}^{n}$ and $y \in \mathbb{R}^{m},(x, y)$ stands for $\left[x^{\mathrm{T}}, y^{\mathrm{T}}\right]^{\mathrm{T}}$. The notation $\mathbb{I}$ denotes the identity mapping from $\mathbb{R}_{\geq 0}$ to $\mathbb{R}_{\geq 0}$. A function $\gamma: \mathbb{R}_{\geq 0} \rightarrow \mathbb{R}_{\geq 0}$ is of class $\mathcal{K}^{-}$if it is continuous, zero at zero and strictly increasing, and is of class $\mathcal{K}_{\infty}$ if, in addition, it is unbounded. A continuous function $\gamma: \mathbb{R}_{\geq 0} \times \mathbb{R}_{\geq 0} \longrightarrow \mathbb{R}_{\geq 0}$ is of class $\mathcal{K} \mathcal{L}$ if for each $t \in \mathbb{R}_{\geq 0}, \gamma(\cdot, t)$ is of class $\mathcal{K}$, and, for each $s \in \mathbb{R}_{>0}, \gamma(s, \cdot)$ is decreasing to zero for $s \rightarrow \infty$. We consider locally Lipschitz Lyapunov functions $U: \mathbb{R}^{n} \rightarrow \mathbb{R}_{>0}$ (that are not necessarily differentiable everywhere) and let $\partial U(x)$ denote the generalised gradient of 
Clarke [27] at a point $x \in \mathbb{R}^{n}$, which is defined as $\partial U(x)=$ $\operatorname{co}\left\{\lim _{i \rightarrow \infty} \nabla U\left(x_{i}\right): x_{i} \rightarrow x\right.$ as $\left.i \rightarrow \infty, x_{i} \notin \Omega_{U}\right\} \quad$ where $\Omega_{U}$ is the union of the set of points where $U$ is not differentiable with any set of Lebesgue-measure zero, and $\operatorname{co}(S)$ stands for the convex hull of the set $S \subset \mathbb{R}^{n}$. For a setvalued mapping $F: \mathbb{R}^{n} \rightrightarrows \mathbb{R}^{m}$, the domain of $F$ is the set $\operatorname{dom} F:=\left\{x \in \mathbb{R}^{n}: F(x) \neq \emptyset\right\}$. Given sets $S_{1}, S_{2} \subset \mathbb{R}^{n}$, let $\left(\begin{array}{l}S_{1} \\ S_{2}\end{array}\right)=S_{1} \times S_{2}$. We let $\mathcal{B}_{s}$, for any $s \in(0, \infty)$, denote the closed ball in $\mathbb{R}^{n}$ centered at the origin with radius $s \in \mathbb{R}_{>0}$. Given a closed set $B \subset \mathbb{R}^{n}$ and $x \in \mathbb{R}^{n}$, the tangent cone at point $x$ to the set $B$ is denoted as $T_{B}(x):=\left\{y: \forall\left\{t_{k}\right\}_{k \in \mathbb{N}_{0}}, t_{k} \downarrow 0, \forall\left\{x_{k}\right\}_{k \in \mathbb{N}_{0}}, x_{k} \rightarrow\right.$ $x$ and $x_{k} \in B, \exists\left\{y_{k}\right\}_{k \in \mathbb{N}_{0}}, y_{k} \rightarrow y$, with $x_{k}+t_{k} y_{k} \in$ $B$ for any $\left.k \in \mathbb{N}_{0}\right\}$. Given a closed set $B_{1} \subset \mathbb{R}^{n} \times \mathbb{R}^{n}$ and points $x, y \in \mathbb{R}^{n}$, let $\|x\|$ denote the Euclidean norm of $x$ and let $\rho_{B_{1}}(x, y):=\inf _{(u, v) \in B_{1}}\|(x-u, y-v)\|$.

We study hybrid systems of the form [20]

$$
\left\{\begin{array}{rlll}
\dot{x} & \in F(x) & & x \in C \\
x^{+} & \in G(x) & & x \in D,
\end{array}\right.
$$

where $x \in \mathbb{R}^{n}$ is the state, $F$ is the flow map, $G$ is the jump map, $C$ is the flow set and $D$ is the jump set. We assume that system (1) satisfies the hybrid basic conditions (see Assumption 6.5 in [20]) given by: (A1) $C$ and $D$ are closed subsets of $\mathbb{R}^{n}$; (A2) $F: \mathbb{R}^{n} \rightrightarrows \mathbb{R}^{n}$ is outer semicontinuous ${ }^{1}$ and locally bounded $^{2}$ relative to $C, C \subset \operatorname{dom} F$, and $F(x)$ is convex for each $x \in C$; (A3) $G: \mathbb{R}^{n} \rightrightarrows \mathbb{R}^{n}$ is outer semicontinuous and locally bounded relative to $D$, and $D \subset \operatorname{dom} G$.

We recall some definitions related to [20]. A subset $E \subset$ $\mathbb{R}_{\geq 0} \times \mathbb{N}_{0}$ is a hybrid time domain if for all $(T, J) \in E, E \cap$ $([0, T] \times\{0,1, \ldots, J\})=\bigcup_{j \in\{0,1, \ldots, J\}}\left(\left[t_{j}, t_{j+1}\right], j\right)$ for some finite sequence of times $0=t_{0} \leq t_{1} \leq \ldots \leq t_{J+1}$. A function $\phi: E \rightarrow \mathbb{R}^{n}$ is a hybrid arc if $E$ is a hybrid time domain and if for each $j \in \mathbb{N}_{0}, t \mapsto \phi(t, j)$ is locally absolutely continuous on $I^{j}:=\{t:(t, j) \in E\}$. The hybrid $\operatorname{arc} \phi: \operatorname{dom} \phi \rightarrow \mathbb{R}^{n}$ is a solution to (1) if: (i) $\phi(0,0) \in C \cup D$; (ii) for any $j \in \mathbb{N}_{0}$, $\phi(t, j) \in C$ and $\frac{d}{d t} \phi(t, j) \in F(\phi(t, j))$ for almost all $t \in I^{j}$; (iii) for every $(t, j) \in \operatorname{dom} \phi$ such that $(t, j+1) \in \operatorname{dom} \phi$, $\phi(t, j) \in D$ and $\phi(t, j+1) \in G(\phi(t, j))$. A solution $\phi$ to (1) is maximal if it cannot be extended and complete if $\operatorname{dom} \phi$ is unbounded. For a solution $\phi$ to (1), $\sup _{t} \operatorname{dom} \phi:=\sup \{t \in$ $\left.\mathbb{R}_{\geq 0}: \exists j \in \mathbb{N}_{0},(t, j) \in \operatorname{dom} \phi\right\}, \sup _{j} \operatorname{dom} \phi:=\sup \{j \in$ $\left.\mathbb{N}_{0}: \exists t \in \mathbb{R}_{\geq 0},(t, j) \in \operatorname{dom} \phi\right\}$, and we call the solution $t$ complete or $j$-complete if $\sup _{t} \operatorname{dom} \phi=\infty$ or $\sup _{j} \operatorname{dom} \phi=$ $\infty$, respectively.

We will use the following stability definitions.

Definition 1. Let $\delta: \mathbb{R}^{n} \rightarrow \mathbb{R}_{\geq 0}$ be continuous. We say that system (1) is

\footnotetext{
${ }^{1}$ The set-valued mapping $F: \mathbb{R}^{n} \rightrightarrows \mathbb{R}^{n}$ is outer semicontinuous if for each $x \in \mathbb{R}^{n}$, every sequence $\left\{x_{i}\right\}_{i \in \mathbb{N}_{0}}$ of points $x_{i} \in \mathbb{R}^{n}, i \in \mathbb{N}_{0}$, convergent to $x$ and any convergent sequence $\left\{y_{i}\right\}_{i \in \mathbb{N}_{0}}$ of points $y_{i} \in F\left(x_{i}\right), i \in \mathbb{N}_{0}$, one has $\lim _{i \rightarrow \infty} y_{i} \in F(x)$, cf. Definition 5.9 in [20].

${ }^{2} \mathrm{~A}$ set-valued mapping $M: \mathbb{R}^{m} \rightrightarrows \mathbb{R}^{n}$ is locally bounded relative to a set $S \subset M$ if, for each $x \in \mathbb{R}^{m}$ there exists a neighbourhood $U_{x}$ of $x$ such that $\left.M\right|_{S}\left(U_{x}\right)$ is bounded, where the set-valued mapping $\left.M\right|_{S}$ from $\mathbb{R}^{m}$ to $\mathbb{R}^{n}$ is defined as $M(x)$ for $x \in S$ and $\emptyset$ for $x \notin S$, see Definition 5.14 in [20]
}

(i) stable with respect to $\delta$ if for any $\varepsilon>0$ there exists $s>0$ such that for any solution $\phi$ to (1) with $\delta(\phi(0,0))<s$, $\delta(\phi(t, j))<\varepsilon$ for any $(t, j) \in \operatorname{dom} \phi$.

(ii) uniformly in $t$ (respectively, in $j$ ) globally pre-attractive with respect to $\delta$ if for any $\varepsilon>0$ and $r>0$, there exists $T>0$ (respectively, $J \in \mathbb{N}_{>0}$ ) such that for any solution $\phi$ to (1) with $\delta(\phi(0,0))<r, \delta(\phi(t, j))<\varepsilon$ for any $(t, j) \in$ dom $\phi$ with $t \geq T$ (respectively, with $j \geq J)$.

(iii) uniformly in $t$ (respectively, in $j$ ) globally preasymptotically stable with respect to $\delta\left(\delta-\mathrm{U}_{\mathrm{t}} \mathrm{GpAS}\right.$ (respectively, $\left.\delta-\mathrm{U}_{\mathrm{j}} \mathrm{GpAS}\right)$ ) if it is both stable with respect to $\delta$ and uniformly in $t$ (respectively, in $j$ ) globally preattractive with respect to $\delta$.

When, in addition all maximal solutions are t-complete (respectively, j-complete), we say that system (1) is uniformly in $t$ (respectively, in $j$ ) globally asymptotically stable with respect to $\delta\left(\delta-\mathrm{U}_{\mathrm{t}} \mathrm{GAS}\right.$ (respectively, $\delta$ - $\left.\mathrm{U}_{\mathrm{j}} \mathrm{GAS}\right)$ ).

The notions of $\delta$ - $\mathrm{U}_{\mathrm{t}} \mathrm{GpAS}$ and $\delta$ - $\mathrm{U}_{\mathrm{j}} \mathrm{GpAS}$ include stability notions of sets by appropriate selection of the function $\delta$.

\section{FROM GRAPHICAL CLOSENESS TO INCREMENTAL ASYMPTOTIC STABILITY}

To define incremental stability for hybrid systems, we need to evaluate the distance between any two solutions of system (1). However, for hybrid systems, two solutions do not have the same hybrid time domain in general. Hence, we may not be able to compare two solutions of a given system at the same (hybrid) time instant. To avoid that issue, we resort to graphical closeness concepts. In particular, the definitions we propose below are inspired by the notion of $\varepsilon$-closeness of hybrid arcs, which is related to the Hausdorff distance between the graphs of the hybrid arcs, see Definition 4.11 in [20].

Definition 2. Given $\varepsilon>0$, two hybrid arcs $\phi_{1}$ and $\phi_{2}$ are $\varepsilon$-close if

(i) for all $(t, j) \in$ dom $\phi_{1}$ there exists $t^{\prime} \in \mathbb{R}_{\geq 0}$ such that $\left(t^{\prime}, j\right) \in \operatorname{dom} \phi_{2},\left|t-t^{\prime}\right|<\varepsilon$ and $\left\|\phi_{1}(t, j)-\phi_{2}\left(t^{\prime}, j\right)\right\|<$ $\varepsilon$.

(ii) for all $(t, j) \in$ dom $\phi_{2}$ there exists $t^{\prime} \in \mathbb{R}_{\geq 0}$ such that $\left(t^{\prime}, j\right) \in \operatorname{dom} \phi_{1},\left|t-t^{\prime}\right|<\varepsilon$ and $\left\|\phi_{2}(t, j)-\phi_{1}\left(t^{\prime}, j\right)\right\|<$ $\varepsilon$.

Using graphical closeness to compare solutions of hybrid systems was motivated by earlier use in [16], [17], [19], [20]. In Definition 2, the hybrid arcs $\phi_{1}$ and $\phi_{2}$ are not compared at the same hybrid time instant but at $(t, j)$ for one and $\left(t^{\prime}, j\right)$ for the other, with $\left|t-t^{\prime}\right|<\varepsilon$. In that way, dom $\phi_{1}$ and dom $\phi_{2}$ do not need to be equal, they only need to be 'close' enough so that for any $(t, j) \in \operatorname{dom} \phi_{1}$ there exists an appropriate pair $\left(t^{\prime}, j\right) \in \operatorname{dom} \phi_{2}$ and vice versa. Definition 2 may therefore be used to compare two solutions to (1), even when these may not have the same hybrid time domain.

The distance between two hybrid arcs is evaluated using the Euclidean distance in Definition 2, which may be restrictive in the context of incremental stability, see [4]. Inspired by [28], we use a generic positive function, which we denote $\delta$, 
instead of the Euclidean distance, to compare the states of two hybrid solutions and we will talk of incremental stability properties with respect to a certain $\delta$, which also allows to characterise 'output' incremental stability (in addition to incremental stability for the full state). We concentrate on mappings $\delta: \mathbb{R}^{n} \times \mathbb{R}^{n} \rightarrow \mathbb{R}_{\geq 0}$, which belong to the set $\mathcal{D}$ of continuous mappings that verify for any $x_{1}, x_{2} \in \mathbb{R}^{n}$ :

(i) $\delta\left(x_{1}, x_{2}\right)=\delta\left(x_{2}, x_{1}\right)$;

(ii) $x_{1}=x_{2} \Rightarrow \delta\left(x_{1}, x_{2}\right)=0$.

The first condition means that $\delta$ is symmetric and the second one states that $\delta$ vanishes when $x_{1}=x_{2}$. In that way, the functions in $\mathcal{D}$ are general enough to encompass the metrics considered in [4], [23] as particular cases and to accommodate the features of state-triggered hybrid systems for which the requirement that $\delta\left(x_{1}, x_{2}\right)=0$ implies $x_{1}=x_{2}$ leads to an overly restrictive stability notion, see [23].

In view of Definition 2 and the discussion above, we propose the following definition of incremental asymptotic stability.

Definition 3. Given $\delta \in \mathcal{D}$, system (1) is uniformly preincrementally asymptotically stable with respect to $\delta$ in the graphical sense ( $\delta$-UpIS) if the following conditions hold:

(i) for any $\varepsilon>0$, there exists $s>0$ such that for any pair of maximal solutions $\left(\phi_{1}, \phi_{2}\right)$ with $\delta\left(\phi_{1}(0,0), \phi_{2}(0,0)\right)<s$ it holds that, for all $(t, j) \in$ dom $\phi_{1}$, there exists $\left(t^{\prime}, j\right) \in$ dom $\phi_{2}$ with $\left|t-t^{\prime}\right|<\varepsilon$ such that $\delta\left(\phi_{1}(t, j), \phi_{2}\left(t^{\prime}, j\right)\right)<$ $\varepsilon$;

(ii) for any $\varepsilon>0$ and $r>0$, there exists $\Theta \geq 0$ such that for any pair of maximal solutions $\left(\phi_{1}, \phi_{2}\right)$ with $\delta\left(\phi_{1}(0,0), \phi_{2}(0,0)\right)<r$ it holds that, for all $(t, j) \in$ dom $\phi_{1}$ with $t+j \geq \Theta$, there exists $\left(t^{\prime}, j\right) \in$ dom $\phi_{2}$ with $\left|t-t^{\prime}\right|<\varepsilon$ such that $\delta\left(\phi_{1}(t, j), \phi_{2}\left(t^{\prime}, j\right)\right)<\varepsilon$.

System (1) is uniformly incrementally asymptotically stable with respect to $\delta$ in the graphical sense ( $\delta$-UIS) when it is $\delta$-UpIS and any maximal solution to $(1)$ is complete.

Item (i) of Definition 3 is a stability property of all maximal solutions. It means that for any $\varepsilon>0$, there exists $s>0$ such that any two maximal solutions $\phi_{1}$ and $\phi_{2}$ are $\varepsilon$-close, in the distance function $\delta$, when $\delta\left(\phi_{1}(0,0), \phi_{2}(0,0)\right)<s$. Item (ii) of Definition 3 is a uniform global attractivity property of all the maximal solutions. It requires that, for any $\varepsilon, r>0$, there exists $\Theta>0$ such that any two maximal solutions $\phi_{1}$ and $\phi_{2}$ with $\delta\left(\phi_{1}(0,0), \phi_{2}(0,0)\right)<r$ are $\varepsilon$-close (in the distance function $\delta$ ) after a uniform amount of time $\Theta$, the 'tails' of the solutions are $\varepsilon$-close. Notice that we do not explicitly state symmetric statements as in Definition 2, as items (i) and (ii) of Definition 3 hold for any pair of maximal solutions.

Remark 1. A related, non-uniform definition for incremental stability of hybrid systems has been provided in [16], [17]. However, uniformity is a key aspect of incremental stability also in continuous-time systems, see [1], we have chosen to provide uniform incremental stability notions by imposing less restrictive conditions on the hybrid time domains of different solutions. As the uniform attractivity requirement in item (ii) of Definition 3 is more restrictive than the eventually closeness requirement of $\phi_{1}$ and $\phi_{2}$ in [16], [17, Example 3.5], the system in this example is not $\delta$-UIS according to Definition 3.

Definition 3 implies that, when there exists a pair of maximal solutions $\phi_{1}$ and $\phi_{2}$ with $\phi_{1}$ complete and $\phi_{2}$ not complete, system (1) can never be $\delta$-UpIS for any $\delta \in \mathcal{D}$, as item (ii) of Definition 3 can never be satisfied. Hence, either all maximal solutions should be complete or all should have a bounded hybrid time domain for the system to be $\delta$-UpIS. Consequently, Definition 3 not only requires the states of any two solutions to remain close and to converge to each other, it also requires their hybrid time domains to be close and to converge to each other (namely, the maximally allowed time mismatch $\left|t-t^{\prime}\right|$ has to decrease when time evolves), which is a strong requirement as confirmed by the proposition below.

Proposition 1. Consider system (1) and suppose it is $\delta$-UIS for a given $\delta \in \mathcal{D}$. Then, one of the following properties holds: (i) $\operatorname{dom} \phi=\mathbb{R}_{\geq 0} \times\{0\}$ for any maximal solution $\phi$; (ii) dom $\phi=$ $\{0\} \times \mathbb{N}_{0}$ for any maximal solution $\phi$.

Proposition 1 implies that, if system (1) is $\delta$-UIS (whatever $\delta \in \mathcal{D}$ is adopted), it is either a purely continuous-time system or a purely discrete-time system, which is clearly restrictive. For this reason, in the following sections, we formulate incremental stability notions, which can be satisfied by a larger class of hybrid systems and enable application of these incremental stability notions to study, e.g., tracking control, observer design or synchronisation problems for hybrid systems. In fact, we present alternative definitions to characterise hybrid systems, which exhibit incremental stability properties with respect to the continuous time, or the discrete time, respectively, which are less restrictive than the generic $\delta$ UIS property proposed in this section. We remark that in [18, Definition 2.2], uniformity of the convergence property is dropped, therewith attaining a less stringent system property in a different manner than proposed here.

Remark 2. Interestingly, hybrid systems with non-complete maximal solutions can be $\delta$-UpIS, while still allowing solutions with both flow and jumps. An example is given by $\dot{x}=-1$ when $x \in[1,2]$ and $x^{+}=0$ when $x=1$, where $x \in \mathbb{R}$ and $\delta$ is the Euclidean distance.

\section{FLOW INCREMENTAL ASYMPTOTIC STABILITY}

Given the restrictive nature of $\delta$-UIS observed in Proposition 1, we present in Section IV-A an incremental stability notion, which considers continuous time as more important than the discrete time. Subsequently, sufficient Lyapunovbased conditions are presented in Section IV-B followed by application of these results for event-triggered control systems in Section IV-C. In Section IV-D, we show how incremental stability can be analysed if 'peaking' of the distance function occurs.

\section{A. Definition}

We propose the next incremental stability definition, in which the solutions to (1) are evaluated at close continuous times, while disregarding the number of jumps elapsed contrary to Definition 3. 
Definition 4. Given $\delta \in \mathcal{D}$, system (1) is flow uniformly pre-incrementally asymptotically stable with respect to $\delta(\delta$ FUpIS) if the following conditions hold:

(i) for any $\varepsilon>0$, there exists $s>0$ such that for any pair of maximal solutions $\left(\phi_{1}, \phi_{2}\right)$ with $\delta\left(\phi_{1}(0,0), \phi_{2}(0,0)\right)<s$ it holds that, for all $(t, j) \in$ dom $\phi_{1}$, there exists $\left(t^{\prime}, j^{\prime}\right) \in$ dom $\phi_{2}$ with $\left|t-t^{\prime}\right|<\varepsilon$ such that $\delta\left(\phi_{1}(t, j), \phi_{2}\left(t^{\prime}, j^{\prime}\right)\right)<$ $\varepsilon ;$

(ii) for any $\varepsilon>0$ and $r>0$, there exists $T \geq 0$ such that for any pair of maximal solutions $\left(\phi_{1}, \phi_{2}\right)$ with $\delta\left(\phi_{1}(0,0), \phi_{2}(0,0)\right)<r$ it holds that, for all $(t, j) \in$ dom $\phi_{1}$ with $t \geq T$, there exists $\left(t^{\prime}, j^{\prime}\right) \in$ dom $\phi_{2}$ with $\left|t-t^{\prime}\right|<\varepsilon$ such that $\delta\left(\phi_{1}(t, j), \phi_{2}\left(t^{\prime}, j^{\prime}\right)\right)<\varepsilon$.

System (1) is flow uniformly incrementally asymptotically stable with respect to $\delta$ ( $\delta$-FUIS) when, in addition, any maximal solution $\phi$ to (1) is t-complete.

Item (i) of Definition 4 is a stability property. It implies that any two solutions $\phi_{1}$ and $\phi_{2}$, which are initialised close to each other (where $\delta$ is used to evaluate the distance between the initial conditions), remain close to each other at some close continuous times, discarding the number of jumps the solutions have experienced. It also implies that $\sup _{t} \operatorname{dom} \phi_{1}$ and $\sup _{t} \operatorname{dom} \phi_{2}$ are 'close', otherwise there may not exist $\left(t^{\prime}, j^{\prime}\right) \in \operatorname{dom} \phi_{2}$ such that $\left|t-t^{\prime}\right|<\varepsilon$ in item (i) of Definition 4. Item (ii) is a uniform global attractivity property of every solution, as the constant $T$ is the same for all maximal solutions $\phi_{1}$ and $\phi_{2}$ with $\delta\left(\phi_{1}(0,0), \phi_{2}(0,0)\right)<r$, given $\varepsilon, r>0$. It can be noted that the time mismatch $t-t^{\prime}$ of the solutions in Definition 4 reminds of Zhukovsky stability for continuous-time systems, see Chapter 8.4 in [29] for instance. If $\delta$ is the Euclidean distance, a small time mismatch $t-t^{\prime}$ can circumvent the 'peaking phenomenon' of the error $\delta\left(\phi_{1}(t, j), \phi_{2}\left(t, j^{\prime}\right)\right)$ as described in e.g., [21], [23], [24].

When there exists a pair of maximal solutions $\phi_{1}$ and $\phi_{2}$ with $\sup _{t} \operatorname{dom} \phi_{1}=\infty$ and $\sup _{t} \operatorname{dom} \phi_{2}<\infty$, the system can never be $\delta$-FUpIS for any $\delta \in \mathcal{D}$, as item (ii) of Definition 4 can never be satisfied. Hence, either all maximal solutions should be $t$-complete or all hybrid time domains should be bounded in the $t$-direction for the system to be $\delta$-FUpIS. In the first case, $\delta$-FUpIS would immediately become $\delta$-FUIS. We also remark that when $\sup _{t} \operatorname{dom} \phi<T^{\prime}<\infty$ for all maximal solutions $\phi$ to (1), with $T^{\prime}>0$, then item (ii) of Definition 4 trivially holds by taking $T=T^{\prime}$.

Remark 3. A formulation of $\delta$-FU(p)IS in Definition 4 can also be given in terms of $\mathcal{K} \mathcal{L}$-functions, similar to [1]. Namely, given $\delta \in \mathcal{D}$, the system (1) is $\delta$-FUpIS if and only if there exists $\beta \in \mathcal{K} \mathcal{L}$ such that for any pair of maximal solutions $\phi_{1}, \phi_{2}$ and any $(t, j) \in \operatorname{dom} \phi_{1}$,

$$
\begin{array}{r}
\inf _{\left(t^{\prime}, j^{\prime}\right) \in \operatorname{dom} \phi_{2}} \max \left(\left|t-t^{\prime}\right|, \delta\left(\phi_{1}(t, j), \phi_{2}\left(t^{\prime}, j^{\prime}\right)\right)\right) \leq \\
\beta\left(\delta\left(\phi_{1}(0,0), \phi_{2}(0,0)\right), t\right)
\end{array}
$$

and (1) is $\delta$-FUIS if and only if, in addition, all maximal solutions $\phi$ are $t$-complete. The only-if-statement trivially holds, whereas the if-statement follows from the observation that the required existence of $\left(t^{\prime}, j^{\prime}\right)$ in items (i) and (ii) of Definition 4 is equivalent to $\inf _{\left(t^{\prime}, j^{\prime}\right) \in \operatorname{dom} \phi_{2}} \max \left(\left|t-t^{\prime}\right|, \delta\left(\phi_{1}(t, j), \phi_{2}\left(t^{\prime}, j^{\prime}\right)\right)\right)<\epsilon$, combined with standard arguments to construct a function $\beta \in \mathcal{K} \mathcal{L}$ from the two mappings $\epsilon \mapsto s(\epsilon)$ and $(\epsilon, r) \mapsto T(\epsilon, r)$ as used e.g. in the proof of Lemma 4.5 in [30].

Remark 4. Definition 4 differs from the two definitions in [18] on several points. First, a solution may experience two consecutive jumps (see Example 1 in [19] for instance) and the maximal solutions to system (1) are not required to be $t$ complete in the definition of $\delta$-UpIS, relaxing [18, Assumption 2.1]. Second, the class of admissible distance-like functions $\mathcal{D}$ is broader than the distance functions allowed in [18]. Third, in contrast to [18], the solutions $\phi_{1}$ and $\phi_{2}$ in Definition 4 are not compared at the same continuous time $t$ but at two (potentially) distinct times $t$ and $t^{\prime}$ with $\left|t-t^{\prime}\right|<\varepsilon$, which provides more flexibility. Fourth, a uniform bound is imposed in Definition 4 on the convergence rate in contrast with [18, Definition 2.2] and [16], [17], but similar to [18, Definition 2.9].

In the following, we propose a Lyapunov characterization of flow incremental stability. We focus on $\delta$-FUIS for any given $\delta \in \mathcal{D}$, and we leave the study of $\delta$-FUpIS for future work.

\section{B. Lyapunov conditions}

We introduce an extended system as suggested in [1] in the context of continuous-time systems and in [31] for hybrid systems (without 'time mismatch'). The idea is to duplicate the system (1). In that way, we are able to compare two solutions of the original system using the extended system, now having the same hybrid time domain. The extended system is given by

$$
\begin{array}{rll}
\left(\dot{x}_{1}, \dot{x}_{2}\right) & \in F_{\mathrm{f}}\left(x_{1}, x_{2}\right) & \left(x_{1}, x_{2}\right) \in C_{\mathrm{f}}, \\
\left(x_{1}^{+}, x_{2}^{+}\right) \in G_{\mathrm{f}}\left(x_{1}, x_{2}\right) & \left(x_{1}, x_{2}\right) \in D_{\mathrm{f}},
\end{array}
$$

where

$$
\begin{aligned}
& C_{\mathrm{f}}:=\left\{\left(x_{1}, x_{2}\right): x_{1} \in C \text { and } x_{2} \in C\right\} \\
& D_{\mathrm{f}}:=\left\{\left(x_{1}, x_{2}\right): x_{1} \in D \text { or } x_{2} \in D\right\},
\end{aligned}
$$

and

$$
\begin{aligned}
& F_{\mathrm{f}}\left(x_{1}, x_{2}\right):=\left(F\left(x_{1}\right), F\left(x_{2}\right)\right) \text { for } x_{1}, x_{2} \in \mathbb{R}^{n} \\
& G_{\mathrm{f}}\left(x_{1}, x_{2}\right):=\left\{\begin{array}{r}
\left(G\left(x_{1}\right),\left\{x_{2}\right\}\right) \\
\text { if } x_{1} \in D \text { and } x_{2} \notin D \\
\left(\left\{x_{1}\right\}, G\left(x_{2}\right)\right) \\
\text { if } x_{1} \notin D \text { and } x_{2} \in D \\
\left\{\left(G\left(x_{1}\right),\left\{x_{2}\right\}\right),\left(\left\{x_{1}\right\}, G\left(x_{2}\right)\right)\right\} \\
\text { if } x_{1} \in D \text { and } x_{2} \in D .
\end{array}\right.
\end{aligned}
$$

The mapping $G_{\mathrm{f}}$ is such that the $x_{1}$-system experiences a jump when $x_{1} \in D$ and vice versa for the $x_{2}$-system. When $x_{1}, x_{2} \in$ $D$, the solution jumps twice in any order. This construction of the jump map is based on [31] and ensures that the jump map (4) is outer semicontinuous, which is one of the hybrid basic conditions, see Section II. In addition, this construction ensures that a solution $\bar{\phi}$ of the extended system (3) has experienced as many jumps as the solutions $\bar{\phi}_{1}$ and $\bar{\phi}_{2}$ together (in contrast to the different definition of the jump map for the extended system proposed in [18]). The next lemma relates solutions to the extended system (3) to solutions to (1). 
Lemma 1. Suppose that any maximal solution to (1) is $t$ complete. The following hold.

(i) Consider any two maximal solutions $\phi_{1}, \phi_{2}$ to (1). There exists a solution $\bar{\phi}$ to (3) such that for each $(t, j) \in$ dom $\phi_{1}$, there exist $\left(t, j^{\prime}\right) \in$ dom $\phi_{2}$ such that

$$
\bar{\phi}\left(t, j+j^{\prime}\right)=\left(\phi_{1}(t, j), \phi_{2}\left(t, j^{\prime}\right)\right) .
$$

(ii) Given any solution $\bar{\phi}$ to (3), there exist two solutions $\phi_{1}, \phi_{2}$ to (1) such that for every $(t, \bar{j}) \in \operatorname{dom} \bar{\phi}$, there exist $(t, j) \in \operatorname{dom} \phi_{1},\left(t, j^{\prime}\right) \in \operatorname{dom} \phi_{2}$ such that $\bar{j}=$ $j+j^{\prime}$, and (6) holds.

Item (i) of Lemma 1 implies that, for any pair of solutions to the original system (1), there exists a maximal solution to the extended system (3), which is equal to the former pair at any continuous time, provided any maximal solution to (1) is $t$-complete. The latter assumption is essential here. Indeed, if the pair $\left(\phi_{1}, \phi_{2}\right)$ of solutions to (1) would be such that $\sup _{t} \operatorname{dom} \phi_{1}$ differs from $\sup _{t} \operatorname{dom} \phi_{2}$, then the solution $\phi$ to the extended system (3) initialized at $\left(\phi_{1}(0,0), \phi_{2}(0,0)\right)$ will not continue past continuous time $\min \left(\sup _{t} \operatorname{dom} \phi_{1}, \sup _{t} \operatorname{dom} \phi_{2}\right)$ and, for this reason, this extended system is not appropriate to characterise $\delta$-FUpIS but it is for $\delta$-FUIS as we show in Theorem 1 below. Item (ii) of Lemma 1 means that, for any solution to (3), there exists a pair of solutions to (1) which, after a change of the discrete times $j$, is mapped onto the solution to (3). Hence, Lemma 1 shows that the solutions to systems (1) and (3) are closely related apart from the discrete times, which are nevertheless irrelevant when investigating flow incremental stability in view of Definition 4. The next result ensures $t$-completeness of all maximal solutions to system (3).

Lemma 2. All maximal solutions $\phi$ to (1) are $t$-complete if and only if all maximal solutions $\bar{\phi}$ to (3) are $t$-complete.

In the following theorem, we characterise $\delta$-FUIS of system (1) in terms of stability properties of the extended system (3). The first characterisation, which we will present in Theorem 1, in fact yields a stronger system property, which is formalised in the next definition. In Section IV-D, we provide a less restrictive characterisation for $\delta$-FUIS.

Definition 5. Given $\delta \in \mathcal{D}$, the system (1) is $t$-matched flow uniformly incrementally asymptotically stable with respect to $\delta$ ( $\delta$-tFUIS) when (1) is $\delta$-UIS and (i) and (ii) of Definition 4 also hold when the requirements $\left|t-t^{\prime}\right|<\epsilon$ are strengthened to $t^{\prime}=t$.

Definition 5 is closely related ${ }^{3}$ to [18, Definition 2.9], such that the following theorem is closely related to Theorem 3.12 of that paper, even though a different extended hybrid system is considered compared to [18].

Theorem 1. Let $\delta \in \mathcal{D}$. The following statements are equivalent: (i) system (3) is $\delta-U_{\mathrm{t}} G A S$, see Definition 1; (ii) system (1) is $\delta$-tFUIS

\footnotetext{
${ }^{3}$ Instead of imposing 'closeness' for all $(t, j) \in \operatorname{dom} \phi_{1}$, in [18, Definition 2.9], only the time instants $\left(t, j_{1}(t)\right)$ are covered, with $j_{1}$ the minimal integer such that $\left(t, j_{1}\right) \in \operatorname{dom} \phi_{1}$, and more generic distance functions are considered here.
}

Theorem 1 shows that $\delta$-tFUIS of system (1) is equivalent to $\delta-\mathrm{U}_{\mathrm{t}} \mathrm{GAS}$ of the extended system (3), similarly to what is done for continuous-time systems in [1]. As a next step, the $\delta$ - $\mathrm{U}_{\mathrm{t}} \mathrm{GAS}$ property of system (3) can be established using the following Lyapunov-based conditions.

Proposition 2. Suppose that there exist $\delta \in \mathcal{D}, U: C_{\mathrm{f}} \cup D_{\mathrm{f}} \cup$ $G_{\mathrm{f}}\left(D_{\mathrm{f}}\right) \rightarrow \mathbb{R}_{>0}$, which is locally Lipschitz on an open set containing $C_{\mathrm{f}} \cup D_{\mathrm{f}} \cup G_{\mathrm{f}}\left(D_{\mathrm{f}}\right), \alpha_{1}, \alpha_{2} \in \mathcal{K}_{\infty}$ and a continuous positive-definite function $\sigma$ such that the following conditions hold:

(i) for any $\left(x_{1}, x_{2}\right) \in C_{\mathrm{f}} \cup D_{\mathrm{f}} \cup G_{\mathrm{f}}\left(D_{\mathrm{f}}\right), \alpha_{1}\left(\delta\left(x_{1}, x_{2}\right)\right) \leq$ $U\left(x_{1}, x_{2}\right) \leq \alpha_{2}\left(\delta\left(x_{1}, x_{2}\right)\right)$;

(ii) for any $\left(x_{1}, x_{2}\right) \in C_{\mathrm{f}}, \zeta \in \partial U\left(x_{1}, x_{2}\right)$, and $f \in$ $F_{\mathrm{f}}\left(x_{1}, x_{2}\right),\langle\zeta, f\rangle \leq-\sigma\left(U\left(x_{1}, x_{2}\right)\right)$

(iii) for any $\left(x_{1}, x_{2}\right) \in D_{\mathrm{f}}$ and $g \in G_{\mathrm{f}}\left(x_{1}, x_{2}\right), U(g) \leq$ $U\left(x_{1}, x_{2}\right)$;

(iv) any maximal solution to (1) is t-complete.

Then system (3) is $\delta-U_{\mathrm{t}} G A S$.

Condition (iii) of Proposition 2 implies that the Lyapunov function should not increase when jumps occur that emanate from the sets $D \times C, C \times D$ and $D \times D$. Our experience is that these conditions are not overly restrictive. In fact, a constructive method to design such Lyapunov functions for a subclass of (piecewise linear) hybrid systems is provided in [32].

The combination of Proposition 2 and Theorem 1 provides Lyapunov-based sufficient conditions for flow incremental stability. We remark that the conditions of Proposition 2 can be relaxed when minimal or maximal (average) inter-jump times can be guaranteed, cf. [32, Theorem 2].

\section{Case study: Event-triggered control}

Consider the plant $\dot{x}=f(x, u)$, where $x \in \mathbb{R}^{n}$ is the state, $u \in \mathbb{R}^{m}$ is the control input and $f: \mathbb{R}^{n} \times \mathbb{R}^{m} \rightarrow \mathbb{R}^{n}$ is continuous. We design the feedback law $u=k(x)$ with $k: \mathbb{R}^{n} \rightarrow \mathbb{R}^{m}$ continuous, which we sample and hold using zero-order-hold devices. Hence, the input applied to the plant is $u=k(\hat{x})$ with $\dot{\hat{x}}=0$ for all $t \in\left(t_{i}, t_{i+1}\right), \hat{x}\left(t_{i}^{+}\right)=x\left(t_{i}\right)$, where $t_{i}, i \in \mathcal{I} \subseteq \mathbb{N}_{0}$, are the sampling instants. The sequence $\left\{t_{i}\right\}_{i \in \mathcal{I}}$ is generated by an event-triggering condition, which means that sampling occurs whenever a state-dependent criterion is satisfied, see [26] for more information. In particular, we consider the rule which triggers a transmission when $\|\hat{x}-x\| \geq \rho$, where $\rho>0$ is a design parameter. This type of triggering law was originally proposed in [33].

The overall system is modeled by the hybrid system

$$
\begin{aligned}
& \left.\begin{array}{rl}
\dot{x} & =f(x, k(x+e)) \\
\dot{e} & =-f(x, k(x+e))
\end{array}\right\} \quad \text { when }\|e\| \leq \rho, \text { and } \\
& \left.x^{+}=x\right\} \\
& \left.e^{+}=0\right\} \\
& \text { when }\|e\| \geq \rho \text {, }
\end{aligned}
$$

where $e:=\hat{x}-x$ denotes the sampling-induced error. System (7) verifies the hybrid basic conditions, see Section II.

We assume that the feedback law $u=k(x)$ ensures the existence of a Lyapunov function for incremental input-to-state stability of the system $\dot{x}=f(x, k(x+e))$, which implies that 
this system is incrementally input-to-state stable, see [1], as formalised in the following assumption.

Assumption 1. There exist a continuously differentiable function $V: \mathbb{R}^{n} \times \mathbb{R}^{n} \rightarrow \mathbb{R}_{\geq 0}, \underline{\alpha}_{V}, \bar{\alpha}_{V}, \gamma \in \mathcal{K}_{\infty}, a_{V}>0$ such that the following conditions hold.

(i) For any $x_{1}, x_{2} \in \mathbb{R}^{n}, \underline{\alpha}_{V}\left(\left\|x_{1}-x_{2}\right\|\right) \leq V\left(x_{1}, x_{2}\right) \leq$ $\bar{\alpha}_{V}\left(\left\|x_{1}-x_{2}\right\|\right)$.

(ii) For any $x_{1}, x_{2}, e_{1}, e_{2} \quad \in \quad \mathbb{R}^{n}$, $\left\langle\nabla V\left(x_{1}, x_{2}\right),\left(f\left(x_{1}, k\left(x_{1}+e_{1}\right)\right), f\left(x_{2}, k\left(x_{2}+e_{2}\right)\right)\right)\right\rangle$ $\leq-a_{V} V\left(x_{1}, x_{2}\right)+\gamma\left(\left\|e_{1}-e_{2}\right\|\right)$.

Assumption 1 can always be ensured when the plant is linear time-invariant, stabilizable and detectable for instance. A nonlinear example is provided at the end of this subsection.

The next proposition states that the event-triggered control system (7) is $\delta$-FUIS with $\delta$ defined as, for any $x_{1}, e_{1}, x_{2}, e_{2} \in$ $\mathbb{R}^{n}$

$$
\delta\left(x_{1}, e_{1}, x_{2}, e_{2}\right)=\max \left\{V\left(x_{1}, x_{2}\right)-a_{V}^{-1} \gamma(2 \rho), 0\right\},
$$

where $V, a_{V}, \gamma$ come from Assumption 1.

Proposition 3. If Assumption 1 holds, then system (7) is $\delta$ FUIS with $\delta$ given in (8).

Proposition 3 means that the incremental (input-to-state) stability property of the continuous-time system $\dot{x}=f(x, k(x))$ guaranteed by Assumption 1 is practically preserved for the event-triggered controlled system (7), in the sense of Definition 4, where the adjustable parameter is $\rho$.

Example 1. Consider the following system, which is similar to the example in Section VI.A in [1]: $\dot{x}_{1}=-\beta x_{1}+\operatorname{sat}\left(x_{2}\right) \operatorname{sat}\left(x_{3}\right), \dot{x}_{2}=-\sigma x_{2}+\sigma x_{3}$ and $\dot{x}_{3}=u$, where $u=-x_{3}, \beta=\frac{8}{3}, \sigma=10$, and $\operatorname{sat}(s)=s$ for $|s| \leq 1$ and $\operatorname{sat}(s)=\frac{s}{|s|}$ for $|s| \geq 1$. The induced system (7) verifies Assumption 1 with $^{4} V\left(x, x^{\prime}\right)=$ $\frac{1}{2}\left(\lambda_{1}\left(x_{1}-x_{1}^{\prime}\right)^{2}+\lambda_{2}\left(x_{2}-x_{2}^{\prime}\right)^{2}+\lambda_{3}\left(x_{3}-x_{3}^{\prime}\right)^{2}\right)$, where $\lambda_{1}=0.0043, \lambda_{2}=0.0017, \lambda_{3}=0.0058$, for any $x=\left(x_{1}, x_{2}, x_{3}\right)$ and $x^{\prime}=\left(x_{1}^{\prime}, x_{2}^{\prime}, x_{3}^{\prime}\right)$, $\underline{\alpha}_{V}(s)=\frac{1}{2} \min _{i \in\{1,2,3\}} \lambda_{i} s^{2}, \bar{\alpha}_{V}(s)=\frac{1}{2} \max _{i \in\{1,2,3\}} \lambda_{i} s^{2}$, $\gamma(s)=0.8674 s^{2}$ for any $s \geq 0$, and $a_{V}=1$. As a result, we can apply Proposition 3 to conclude that the event-triggered control implementation of the feedback law $u=-x_{3}$ ensures that the corresponding system (7) is $\delta$-FUIS with $\delta$ given in (8) for any $\rho>0$.

\section{Flow incremental stability with time mismatch}

The Lyapunov conditions of flow incremental stability in Section IV-B imply that the distance $\delta$ between solutions decreases when compared at the same continuous-time instant, i.e. no time mismatch is needed and $\delta$-tFUIS is shown. When $\delta$ is the Euclidean distance, system (1) may be $\delta$-FUIS but not $\delta$ tFUIS because of the 'peaking phenomenon', see, e.g., [23] in the context of tracking control. To study $\delta$-FUIS in this case, we may resort to an auxiliary distance function $\rho_{\mathcal{A}}$ defined below, which overcomes this issue, such that $\rho_{\mathcal{A}}$-tFUIS may

${ }^{4}$ The values of $\lambda_{1}, \lambda_{2}, \lambda_{3}$ and $\gamma$ were obtained using YALMIP [34]. be established (using the results in Section IV-B) leading to $\delta$-FUIS. Indeed, the theorem stated below provides conditions when $\rho_{\mathcal{A}}$-tFUIS implies $\delta$-FUIS

To construct such $\rho_{\mathcal{A}}$ motivated by [23], [32], we define $\mathcal{A}:=\left\{\begin{array}{r}\left(x_{1}, x_{2}\right) \in(C \cup D \cup G(D))^{2}: \exists k_{1}, k_{2} \in \mathbb{N}_{0}, \\ \bar{G}^{k_{1}}\left(x_{1}\right) \cap \bar{G}^{k_{2}}\left(x_{2}\right) \neq \emptyset\end{array}\right\}$, where $\bar{G}(x):=G(x)$ for $x \in D$ and $\bar{G}(x):=\emptyset$ for all $x \notin D, \bar{G}^{k+1}(x)$ is inductively defined with $\bar{G}^{k+1}(x)=$ $\bar{G}\left(\bar{G}^{k}(x)\right)$ and $\bar{G}(x)^{0}=x$. We consider the distance function $\rho_{\mathcal{A}}\left(x_{1}, x_{2}\right)=\inf _{\left(y_{1}, y_{2}\right) \in \mathcal{A}}\left\|\left(x_{1}-y_{1}, x_{2}-y_{2}\right)\right\|$, which clearly satisfies $\rho_{\mathcal{A}} \in \mathcal{D}$. When $\mathcal{A}$ is an invariant set to (3) (this condition can hold while invariance of the set of points where $x_{1}=x_{2}$ is not, see e.g. [23]), we can expect that solutions starting nearby $\mathcal{A}$ will at least stay close to it over jumps, even though they may be diverge from the set $\mathcal{A}$ during flows. Consequently, no 'peaking phenomenon' is expected in the distance $\rho_{\mathcal{A}}$ when two solutions converge towards each other, see [23]. The next theorem shows that $\rho_{\mathcal{A}}$-tFUIS implies $\delta$ FUIS, where we recall that $T_{C}(x)$ is the tangent cone to $C$ at $x$, see Section II.

Theorem 2. Consider system (1), let $\delta$ be the Euclidean distance and suppose that the following hold:

(i) $G(D) \cap D=\emptyset$ and $G$ is single-valued;

(ii) $\forall x \in C \cap D, \quad F(x) \cap T_{C}(x)=\emptyset$;

(iii) $\forall x \in C \cap G(D),-F(x) \cap T_{C}(x)=\emptyset$;

(iv) $D$ is bounded.

If system (1) is $\rho_{\mathcal{A}}$-tFUIS, then it is $\delta$-FUIS.

Property (ii) of Theorem 2 (combined with (i) and (iv)) implies that when a solution $\phi$ is located in a small neighbourhood of $D$ at hybrid time $(t, j)$, then it will experience a jump at time $\left(t^{\prime \prime}, j\right)$ with $\left|t-t^{\prime \prime}\right|$ small. This observation is exploited to construct the times $\left(t^{\prime}, j^{\prime}\right)$ as in Definition 4, when $t^{\prime}>t$ is selected. The cases where $t^{\prime}<t$ holds are investigated by extending solutions backward in time and exploit property (iii).

The combination of conditions (i) and (iv) in Theorem 2 implies that solutions to (1) will satisfy a minimal inter-jump time and greatly simplifies the geometry of the set $\mathcal{A}$; in particular, these imply that $\mathcal{A} \backslash\{(x, y): x=y \in C \cup D\}$ is a compact set, which is exploited in the proof of this theorem. We expect that these conditions can be relaxed. Conditions (ii) and (iii) of Theorem 2 imply that solutions to (1) cannot both flow and jump from the same point in the state space, and the same holds for the solution in the backward direction of time.

Remark 5. Conditions (ii)-(iv) of Theorem 2, as well as the selection of $\delta$ as the Euclidean distance, are exploited to find a uniform bound on the time-mismatch between the jumps of two solutions. If $D \cap C$ is a smooth manifold, such a bound could also be obtained by requiring that all solutions to the differential inclusion $\dot{x} \in F(x)$ traverse this manifold transversally. In this manner, unbounded $D$ and other functions $\delta \in \mathcal{D}$ could also be considered.

\section{JUMP INCREMENTAL ASYMPTOTIC STABILITY}

The notion of incremental stability presented in Section IV concentrates on the incremental behavior of solutions along 
the continuous-time axis and ignores the number of jumps the solutions have experienced. We propose in this section a symmetric definition emphasizing the discrete time, while ignoring the amount of continuous time during which two solutions have been flowing so far.

\section{A. Definition}

Similar to flow incremental asymptotic stability, we define below the symmetric notion of jump incremental asymptotic stability.

Definition 6. Given $\delta \in \mathcal{D}$, system (1) is jump uniformly preincrementally asymptotically stable with respect to $\delta$ ( $\delta$-JUpIS) if the following conditions hold:

(i) for any $\varepsilon>0$, there exists $s>0$ such that for any pair of maximal solutions $\left(\phi_{1}, \phi_{2}\right)$ with $\delta\left(\phi_{1}(0,0), \phi_{2}(0,0)\right)<s$ it holds that, for all $(t, j) \in$ dom $\phi_{1}$, there exists $\left(t^{\prime}, j\right) \in$ dom $\phi_{2}$ such that $\delta\left(\phi_{1}(t, j), \phi_{2}\left(t^{\prime}, j\right)\right)<\varepsilon$;

(ii) for any $\varepsilon>0$ and $r>0$, there exists $J \geq 0$ such that for any pair of maximal solutions $\left(\phi_{1}, \phi_{2}\right)$ with $\delta\left(\phi_{1}(0,0), \phi_{2}(0,0)\right)<r$ it holds that, for all $(t, j) \in$ dom $\phi_{1}$ with $j \geq J$, there exists $\left(t^{\prime}, j\right) \in$ dom $\phi_{2}$ such that $\delta\left(\phi_{1}(t, j), \phi_{2}\left(t^{\prime}, j\right)\right)<\varepsilon$.

System (1) is jump uniformly incrementally asymptotically stable with respect to $\delta$ ( $\delta$-JUIS) when, in addition, any maximal solution $\phi$ to (1) is $j$-complete.

In item (i) of Definition 6, the distance between two solutions is evaluated at the discrete time $j$, without imposing any conditions on the continuous time in contrast with Definition 4. It has to be noted that the solutions $\phi_{1}$ and $\phi_{2}$ in items (i) and (ii) of Definition 6 are evaluated at the same discrete time $j$, and not at (different) $j$ and $j^{\prime}$, respectively, with $\left|j-j^{\prime}\right|<\varepsilon$ as the reader might expect. That is justified by the fact that when $\varepsilon<1,\left|j-j^{\prime}\right|<\varepsilon$ implies $j=j^{\prime}$ since $j, j^{\prime} \in \mathbb{N}_{0}$. Since the satisfaction of items (i) and (ii) of Definition 6 for any $\varepsilon \in(0,1)$ implies their satisfaction for any $\varepsilon \geq 1$, there is no loss of generality in evaluating $\phi_{1}$ and $\phi_{2}$ at the same discrete time $j$. We emphasise again that item (ii) of Definition 6 is a uniform attractivity property, as the constant $J$ is the same for all maximal solutions $\phi_{1}$ and $\phi_{2}$ with $\delta\left(\phi_{1}(0,0), \phi_{2}(0,0)\right)<r$, given $\varepsilon, r>0$.

Similar observations as for Definition 4 can be made. For instance, when there exists a pair of maximal solutions $\left(\phi_{1}, \phi_{2}\right)$ with $\sup _{j} \operatorname{dom} \phi_{1}=\infty$ and $\sup _{j} \operatorname{dom} \phi_{2}<\infty$, the system can never be $\delta$-JUpIS for any $\delta \in \mathcal{D}$, which implies that either all maximal solutions are $j$-complete or all have a time domain that is bounded in the $j$-direction for the system to be $\delta$-JUpIS.

Remark 6. The existence of $\left(t^{\prime}, j\right)$ as in Definition 6 implies $\inf _{\left(t^{\prime}, j\right) \in \operatorname{dom} \phi_{2}} \delta\left(\phi_{1}(t, j), \phi_{2}\left(t^{\prime}, j\right)\right)<\epsilon$. We then derive that the formulation of $\delta$-JU(p)IS in Definition 6 can be formulated in terms of $\mathcal{K} \mathcal{L}$-functions. Namely, given $\delta \in \mathcal{D}$, the system (1) is $\delta$-JUpIS if and only if there exists $\beta \in \mathcal{K} \mathcal{L}$ such that for any pair $\left(\phi_{1}, \phi_{2}\right)$ of maximal solutions and any $(t, j) \in \operatorname{dom} \phi_{1}$,

$\inf _{\left(t^{\prime}, j\right) \in \operatorname{dom} \phi_{2}} \delta\left(\phi_{1}(t, j), \phi_{2}\left(t^{\prime}, j\right)\right) \leq \beta\left(\delta\left(\phi_{1}(0,0), \phi_{2}(0,0)\right), j\right)$ holds and (1) is $\delta$-JUIS if and only if, in addition, all maximal solutions $\phi$ are $j$-complete.

\section{B. Lyapunov conditions}

To provide conditions for jump incremental stability, similar to the results in Section IV-B for flow incremental stability, we define an extended hybrid system and relate jump incremental stability of (1) to a uniform asymptotic stability property of the extended system. We first introduce the function $\bar{F}(x)=F(x)$ when $x \in C$ and $\bar{F}(x)=\emptyset$ otherwise.

In analogy to (3), we define the hybrid system

$$
\begin{array}{llll}
\left(\dot{x}_{1}, \dot{x}_{2}\right) & \in F_{\mathrm{j}}\left(x_{1}, x_{2}\right) & \left(x_{1}, x_{2}\right) \in C_{\mathrm{j}}, \\
\left(x_{1}^{+}, x_{2}^{+}\right) & \in & G_{\mathrm{j}}\left(x_{1}, x_{2}\right) & \left(x_{1}, x_{2}\right) \in D_{\mathrm{j}},
\end{array}
$$

where

$$
\begin{array}{ll}
C_{\mathrm{j}} & :=(C \times C) \cup(C \times D) \cup(D \times C), \\
D_{\mathrm{j}} & :=D \times D, \\
F_{\mathrm{j}}\left(x_{1}, x_{2}\right) & :=\operatorname{co}\left\{\left(\bar{F}\left(x_{1}\right), 0\right) \times\left(0, \bar{F}\left(x_{2}\right)\right)\right\}, \\
G_{\mathrm{j}}\left(x_{1}, x_{2}\right) & :=\left(G\left(x_{1}\right), G\left(x_{2}\right)\right),
\end{array}
$$

for $x_{1}, x_{2} \in \mathbb{R}^{n}$. The $x_{1}$ - and $x_{2}$-subsystems are essentially copies of system (1). To study JUIS, however, the jumps of the corresponding solutions have to be synchronised. This motivates the construction of $F_{\mathrm{j}}$, that allows flow for either subsystem whenever possible, but when it has reached $D$ and can no longer flow, it 'waits' (the flow map is zero for this subsystem), until the other subsystem also reaches $D$. Subsequently, both subsystems can jump in synchrony following $G_{\mathrm{j}}$. The convex hull in the construction of $F_{\mathrm{j}}$ ensures that the (10) verifies the hybrid basic conditions. The relation between solutions to (10) and (1) are provided in the following two lemmas.

\section{Lemma 3. The following statements hold.}

(i) Consider any two $j$-complete solutions $\phi_{1}, \phi_{2}$ to (1). There exists a solution $\bar{\phi}$ to (10) such that for every $(t, j) \in \operatorname{dom} \phi_{1}$, there exists $\left(t^{\prime}, j\right) \in$ dom $\phi_{2}$ such that

$$
\bar{\phi}\left(t+t^{\prime}, j\right)=\left(\phi_{1}(t, j), \phi_{2}\left(t^{\prime}, j\right)\right) \text {. }
$$

(ii) Given any solution $\bar{\phi}$ to (10), there exist two solutions $\phi_{1}, \phi_{2}$ to (1) such that for every $(\bar{t}, j) \in \operatorname{dom} \bar{\phi}$, there exist $(t, j) \in \operatorname{dom} \phi_{1}$ and $\left(t^{\prime}, j\right) \in \operatorname{dom} \phi_{2}$, such that $t+t^{\prime}=\bar{t}$ and (12) holds.

Lemma 4. All maximal solutions $\phi$ to (1) are $j$-complete if and only if all maximal solutions $\bar{\phi}$ to $(10)$ are $j$-complete.

Analogously to our analysis of $\delta$-FUIS of system (1) in Section IV-B, we characterise $\delta$-JUIS in terms of stability properties of the extended system (10).

Theorem 3. Let $\delta \in \mathcal{D}$. The following statements are equivalent: (i) System (10) is $\delta$ - $U_{\mathrm{j}} G A S$; (ii) System (1) is $\delta$-JUIS.

We now present Lyapunov-based conditions for the latter system property, which, by Theorem 3 , also provides sufficient conditions for $\delta$-JUIS.

Proposition 4. Suppose that there exist $\delta \in \mathcal{D}, U: C_{\mathrm{j}} \cup D_{\mathrm{j}} \cup$ $G_{\mathrm{j}}\left(D_{\mathrm{j}}\right) \rightarrow \mathbb{R}_{\geq 0}$, which is locally Lipschitz on an open set 
containing $C_{\mathrm{j}} \cup D_{\mathrm{j}} \cup G_{\mathrm{j}}\left(D_{\mathrm{j}}\right), \alpha_{1}, \alpha_{2} \in \mathcal{K}_{\infty}$, and a continuous positive-definite function $\sigma$ such that the following hold:

(i) for any $\left(x_{1}, x_{2}\right) \in C_{\mathrm{j}} \cup D_{\mathrm{j}} \cup G_{\mathrm{j}}\left(D_{\mathrm{j}}\right), \alpha_{1}\left(\delta\left(x_{1}, x_{2}\right)\right) \leq$ $U\left(x_{1}, x_{2}\right) \leq \alpha_{2}\left(\delta\left(x_{1}, x_{2}\right)\right)$;

(ii) for any $\left(x_{1}, x_{2}\right) \in C_{\mathrm{j}}, \zeta \in \partial U\left(x_{1}, x_{2}\right)$, and $f \in$ $F_{\mathrm{j}}\left(x_{1}, x_{2}\right),\langle\zeta, f\rangle \leq 0$

(iii) for any $\left(x_{1}, x_{2}\right) \in D_{\mathrm{j}}$ and $g \in G_{\mathrm{j}}\left(x_{1}, x_{2}\right), U(g)-$ $U\left(x_{1}, x_{2}\right) \leq-\sigma\left(U\left(x_{1}, x_{2}\right)\right)$

(iv) any maximal solution to (1) is $j$-complete.

Then system (10) is $\delta-U_{\mathrm{j}} G A S$.

It is possible to relax the conditions of Proposition 4 when solutions to (10) satisfy a persistent jump condition [20], or minimal or maximal (average) inter-jump time properties, cf. [32].

\section{Example: Bouncing ball}

Consider the bouncing ball system with state $x=(p, v)$, where $p$ is the position and $v$ is the velocity, $F(x)=$ $\{(v,-g)\}, G(x)=\{-\varepsilon x\}, C=[0, \infty) \times \mathbb{R}, D=\{0\} \times$ $(-\infty, 0]$, with $\varepsilon \in(0,1)$ the restitution coefficient and constant $g>0$ denoting the gravitational acceleration.

Let $E(x)=\frac{1}{2} v^{2}+g p$ denotes the sum of the kinetic and potential energy for a ball with state $x$. We define the distance function $\delta$ used to investigate jump incremental stability as

$$
\delta\left(x_{1}, x_{2}\right)=\left|E\left(x_{1}\right)-E\left(x_{2}\right)\right|,
$$

for any $x_{1}, x_{2} \in C \cup D$ and note that $\delta$ belongs to $\mathcal{D}$.

We now verify that the conditions of Proposition 2. Define $U\left(x_{1}, x_{2}\right)=\frac{1}{2} \delta^{2}\left(x_{1}, x_{2}\right)=\frac{1}{2}\left(E\left(x_{1}\right)-E\left(x_{2}\right)\right)^{2}$ for $x_{1}, x_{2} \in$ $\mathbb{R}$, such that item (i) of Proposition 4 holds with $\alpha_{1}(s)=$ $\alpha_{2}(s)=\frac{1}{2} s^{2}$ and $U$ is locally Lipschitz. With $F_{j}\left(x_{1}, x_{2}\right)=$ $\left\{\left(\beta v_{1},-\beta g,(1-\beta) v_{2},-(1-\beta) g\right): \beta \in[0,1]\right\}$, we find, for $\zeta \in \partial U$

$$
\begin{array}{r}
\langle\partial U, f\rangle=\left(E\left(x_{1}\right)-E\left(x_{2}\right)\right)\left(g, v_{1},-g,-v_{2}\right)^{T} \\
\left(\beta v_{1},-\beta g,(1-\beta) v_{2},-(1-\beta) g\right)=0
\end{array}
$$

for any $f \in F_{j}\left(x_{1}, x_{2}\right)$ and $x \in C_{j}$, since $\partial U=\left\{\left(E\left(x_{1}\right)-\right.\right.$ $\left.\left.E\left(x_{2}\right)\right)\left(g, v_{1},-g,-v_{2}\right)^{T}\right\}$. Hence, item (ii) of Proposition 4 is satisfied. Let $x_{1}, x_{2} \in D_{j}$. By definition of the jump set and jump map, $U\left(G_{j}\left(x_{1}, x_{2}\right)\right)=U\left(G\left(x_{1}\right), G\left(x_{2}\right)\right)=\frac{1}{4}\left(\left(\varepsilon v_{1}\right)^{2}-\right.$ $\left.\left(\varepsilon v_{2}\right)^{2}\right)^{2}=\varepsilon^{4} U\left(x_{1}, x_{1}\right)$. Hence, item (iii) of Proposition 4 holds with $\sigma(s)=\left(1-\epsilon^{4}\right) s$ for $s \geq 0$.

Finally, all maximal solutions $\phi$ to the bouncing ball system are complete and Zeno as observed in [20], these are $j$ complete. Hence, Proposition 4 proves that the extended hybrid system (10) is $\delta$ - $\mathrm{U}_{\mathrm{j}} \mathrm{GAS}$. With Theorem 3, we conclude that the bouncing ball system is $\delta$-JUIS.

Remark 7. With this design of $\delta$, we have proved incremental stability of the Poincaré return map (cf. [30]) with the Poincaré section taken at $D$.

\section{RELATIONS BETWEEN THE DEFINITIONS}

In this section, we analyze the relations between Definitions 3 , 4, and 6. First, a system, which is $\delta$-FU(p)IS, is not necessarily $\delta$-JU(p)IS and vice versa, as demonstrated by the next two examples.
Example 2 ( $\delta$-FUIS but not $\delta$-JUpIS). Consider the hybrid systems, with parameters $\rho>0$ and $N \in \mathbb{N}_{>0}$, given by

$$
\begin{aligned}
(\dot{x}, \dot{\sigma}) & \in(-x,[0, \rho]) & & (x, \sigma) \in C \\
\left(x^{+}, \sigma^{+}\right) & =(\min \{x, 1\}, \sigma-1) & & (x, \sigma) \in D,
\end{aligned}
$$

where $C=\{(x, \sigma): x \in[0,1]$ and $\sigma \in[0, N]\}$ and $D=$ $\{(x, \sigma): x \in[1, \infty)$ and $\sigma \in[1, N]\}$. This system is $\delta$-FUIS with $\delta:\left(x_{1}, \sigma_{1}, x_{2}, \sigma_{2}\right) \mapsto\left\|x_{1}-x_{2}\right\|$, see Example 1 in [19] for a proof. Nonetheless, it cannot be $\delta$-JUpIS as some maximal solutions are $j$-complete (consider those for which $\dot{\sigma}=\frac{\rho}{2}$ for instance) and some have a time domain that is bounded in the $j$-direction (when $\sigma$ remains constant on flows). As a consequence, item (i) of Definition 6 does not hold.

Example 3 ( $\delta$-JUIS but not $\delta$-FUpIS). Consider the system $\dot{x}=-1$ when $x \in[1, \infty)$ and $x^{+}=\frac{1}{2} x$ when $x \in[0,1]$, which is JUIS with respect to the Euclidean distance according to Example 2 in [19], and suppose, in order to attain a contradiction, that it is FUpIS with respect to the Euclidean distance. As a consequence, for $r>1$ and $\varepsilon \in\left(0, \frac{r}{2}\right)$, there exists $T \geq 0$ such that the statement in item (ii) of Definition 4 holds. Let $\phi_{1}$ and $\phi_{2}$ be two maximal solutions with $\phi_{1}(0,0)=\left(\alpha+\frac{1}{2}\right) r$ and $\phi_{2}(0,0)=\alpha r$ where $\alpha>1$ is a parameter we are free to select. We see that $\left\|\phi_{1}(0,0)-\phi_{2}(0,0)\right\|=\frac{r}{2}<r$. Moreover, since $\alpha r>1$, $\operatorname{dom} \phi_{i}=\left(\left[0, \phi_{i}(0,0)-1\right] \times\{0\}\right) \cup\left(\left\{\phi_{i}(0,0)-1\right\} \times \mathbb{N}_{>0}\right)$ for $i \in\{1,2\}$. We select $\alpha$ sufficiently large such that $\phi_{1}(0,0)-1=\left(\alpha+\frac{1}{2}\right) r-1 \geq T$. Let $t=\phi_{1}(0,0)-1$ and $j \in \mathbb{N}_{0}$ be such that $(t, j) \in \operatorname{dom} \phi_{1}$. According to item (ii) of Definition 4 , there exists $\left(t^{\prime}, j^{\prime}\right) \in \operatorname{dom} \phi_{2}$ such that $\left|t-t^{\prime}\right|<\varepsilon$. Note that $t^{\prime} \leq \phi_{2}(0,0)-1$ by definition of $\operatorname{dom} \phi_{2}$. Consequently, $\left|t-t^{\prime}\right|=\phi_{1}(0,0)-1-t^{\prime} \geq \phi_{1}(0,0)-\phi_{2}(0,0)$. We deduce that $\frac{r}{2}=\phi_{1}(0,0)-\phi_{2}(0,0) \leq\left|t-t^{\prime}\right|<\varepsilon$. This contradicts the fact that $\varepsilon \in\left(0, \frac{r}{2}\right)$. As a consequence, the system is not FUpIS with respect to the Euclidean distance, although it is JUIS with respect to this distance.

The proposition below shows the connections between Definition 3 and Definitions 4-6.

Proposition 5. Let $\delta \in \mathcal{D}$. The following statements hold.

(i) If system (1) is $\delta$-UpIS, then it is both $\delta$-FUpIS and $\delta$-JUpIS.

(ii) If system (1) is $\delta$-UIS, then it is either $\delta$-FUIS or $\delta$-JUIS.

(iii) If system (1) is both $\delta$-FUpIS and $\delta$-JUpIS, it is not necessarily $\delta$-UpIS.

Item (iii) of Proposition 5 is due to the fact that the hybrid time domains of the solutions play a very important role for $\delta$-UIS. Indeed, a system may very well be both $\delta$-FUpIS and $\delta$-JUpIS, and not $\delta$-UpIS (for some $\delta \in \mathcal{D}$ ), because two (maximal) solutions, which have close initial conditions according to the distance $\delta$ do not have 'close' hybrid time domains.

\section{CONSISTENCY WITH DEFINITIONS FOR CONTINUOUS-TIME AND DISCRETE-TIME SYSTEMS}

The proposition below shows that the proposed definitions are consistent with the definitions of incremental stability available in the literature for continuous-time systems. 
Proposition 6. Consider the continuous-time system $\dot{x} \in$ $F(x)$, where $x \in \mathbb{R}^{n}$, and $F: \mathbb{R}^{n} \rightrightarrows \mathbb{R}^{n}$ is outer semicontinuous and locally bounded on $\mathbb{R}^{n}$, and $F(x)$ is convex for each $x \in \mathbb{R}^{n}$. Suppose that any maximal solution is complete and that there exist $\delta \in \mathcal{D}$ and $\beta \in \mathcal{K} \mathcal{L}$ such that any pair of maximal solutions $\left(x_{1}, x_{2}\right)$ verifies for all $t \geq 0$

$$
\delta\left(x_{1}(t), x_{2}(t)\right) \leq \beta\left(\delta\left(x_{1}(0), x_{2}(0)\right), t\right) .
$$

Then the hybrid system (1) with $C=\mathbb{R}^{n}, G(x)=\{x\}$ and $D=\emptyset$, for $x \in \mathbb{R}^{n}$, is $\delta$-FUIS and $\delta$-UIS.

Proposition 6 states that if a continuous-time system is incrementally stable in the sense that (16) holds ${ }^{5}$, then this property is preserved when this system is embedded as a hybrid system of the form (1). Note that the choice of $G$ in Proposition 6 has no impact on the result.

The following proposition states an equivalent result for discrete-time systems. Incremental stability of discrete-time systems is investigated in [25] for instance.

Proposition 7. Consider the discrete-time system $x^{+} \in G(x)$, where $x \in \mathbb{R}^{n}, G: \mathbb{R}^{n} \rightrightarrows \mathbb{R}^{n}$ is outer semicontinuous and locally bounded on $\mathbb{R}^{n}$, and nonempty for all $x \in \mathbb{R}^{n}$. Suppose that this system is incrementally asymptotically stable with respect to $\delta \in \mathcal{D}$, in the sense that there exist $\beta \in \mathcal{K} \mathcal{L}$ such that any pair of maximal solutions $\left(x_{1}, x_{2}\right)$ verifies for all $k \in \mathbb{N}_{0}$

$$
\delta\left(x_{1}(k), x_{2}(k)\right) \leq \beta\left(\delta\left(x_{1}(0), x_{2}(0)\right), k\right) .
$$

Then the hybrid system (1) with $F(x)=\{x\}, C=\emptyset$, and $D=\mathbb{R}^{n}$, for $x \in \mathbb{R}^{n}$, is $\delta$-JUIS and $\delta$-UIS.

\section{CONCLUSIONS}

We have proposed definitions of uniform incremental stability for hybrid systems based on the graphical closeness of solutions, which are applicable both to complete and non-complete solutions. In this context, defining incremental stability with respect to the hybrid time appeared to be very restrictive, motivating two alternative incremental stability notions. The latter can be seen as closeness of the graphs of hybrid solutions when the time domain is projected onto either the continuoustime domain or the discrete-time domain, respectively. Hence, these definitions are relevant in hybrid systems where either the continuous time or the discrete time is dominant. We have investigated the relationship between the presented definitions and showed that they are consistent with the definitions for incremental stability for continuous-time and discrete-time systems.

By introducing extended systems whose solutions capture any pair of solutions and keeping either the continuous time or discrete time synchronised, we enable the usage of setstability results to investigate incremental stability. We have then presented Lyapunov-based sufficient conditions for both incremental stability notions in terms of these extended systems. Various examples are given that illustrate the merits of

${ }^{5}$ Property (16) generalises the definition in [1] to non-Euclidean functions $\delta$, cf. [4]. the incremental stability definitions as well as the Lyapunovbased sufficient conditions. In particular, a case study on eventtriggered control shows the applicability of our findings for systems where continuous time is dominant, and using the bouncing ball system with Zeno-behaviour, we have illustrated the definition and Lyapunov conditions for incremental stability when discrete time is most prominent.

Including time-varying input signals in hybrid systems and investigating incremental stability and incremental input-tostate stability for such systems is subject to future research. We are convinced that the present study provides a key stepping stone to investigate incremental stability for such systems as well. Given the successful application of incremental stability theory for continuous-time and discrete-time systems, we expect that the presented results provide essential tools to address e.g. synchronisation, tracking control and observer design problems for hybrid systems.

\section{REFERENCES}

[1] D. Angeli, "A Lyapunov approach to incremental stability properties," IEEE Transactions on Automatic Control, vol. 47, no. 3, pp. 410-421, 2002.

[2] V. Fromion, S. Monaco, and D. Normand-Cyrot, "A link between inputoutput stability and Lyapunov stability," Systems \& Control Letters, vol. 27, pp. 243-248, 1996.

[3] B. Rüffer, N. van de Wouw, and M. Mueller, "Convergent systems vs. incremental stability," Syst. \& Control Letters, vol. 62, no. 3, pp. 277 285,2013

[4] M. Zamani and P. Tabuada, "Backstepping design for incremental stability," IEEE Transactions on Automatic Control, vol. 56, no. 9, pp. 2184-2189, 2011.

[5] A. Pavlov, N. van de Wouw, and H. Nijmeijer, Uniform output regulation of nonlinear systems: a convergent dynamics approach. Birkhäuser, 2006.

[6] F. Forni and R. Sepulchre, "A differential Lyapunov framework for contraction analysis," IEEE Transactions on Automatic Control, vol. 59, pp. 614-628, 2014.

[7] A. Pogromsky, "Passivity based design of synchronizing systems," International Journal of Bifurcation and Chaos, vol. 8, pp. 295-319, 1998.

[8] N. van de Wouw and A. Pavlov, "Tracking and synchronisation for a class of pwa systems," Automatica, vol. 44, pp. 2909-2915, 2008.

[9] D. C. Tarraf, A. Megretski, and M. A. Dahleh, "A framework for robust stability of systems over finite alphabets," IEEE Transaction on Automatic Control, vol. 53, no. 5, pp. 1133-1146, 2008.

[10] J. H. Richter, W. P. M. H. Heemels, N. van de Wouw, and J. Lunze, "Reconfigurable control of piecewise affine systems with actuator and sensor faults: stability and tracking," Automatica, vol. 47, no. 4, pp. 678-691, 2011.

[11] A. Pavlov, N. van de Wouw, and H. Nijmeijer, "Frequency response functions for nonlinear convergent systems," IEEE Transactions on Automatic Control, vol. 52, no. 6, pp. 1159 - 1165, 2007.

[12] B. Besselink, N. van de Wouw, J. M. A. Scherpen, and H. Nijmeijer, "Model reduction for nonlinear systems by incremental balanced truncation," IEEE Transactions on Automatic Control, vol. 59, no. 10, pp. 2739-2753, 2014.

[13] G. Pola, A. Girard, and P. Tabuada, "Approximately bisimilar symbolic models for incrementally stable switched systems," IEEE Transactions on Automatic Control, vol. 55, no. 1, pp. 116-126, 2010.

[14] D. Fiore, S. J. Hogan, and M. D. Bernardo, "Contraction analysis of switched systems via regularization," Automatica, vol. 73, pp. 279-288, 2016.

[15] V. A. Yakubovich, "Matrix inequalities method in stability theory for nonlinear control systems: I. absolute stability of forced vibrations," Automat. Remote Control, vol. 7, pp. 905-917, 1998.

[16] Y. Li and R. G. Sanfelice, "On necessary and sufficient conditions for incremental stability of hybrid systems using the graphical distance between solutions," in IEEE Conference on Decision and Control, Osaka, Japan, 2015, pp. 5575-5580. 
[17] — , "Incremental graphical asymptotic stability for hybrid dynamical systems," ser. Feedback Stabilization of Controlled Dynamical Systems - Lecture Notes in Control and Information Sciences. Springer, March 2017, vol. 473, pp. 231-262

[18] Y. Li, S. Phillips, and R. G. Sanfelice, "Basic properties and characterizations of incremental stability prioritizing flow time for a class of hybrid systems," Systems \& Control Letters, vol. 90, pp. 7-15, 2016.

[19] R. Postoyan, J. J. B. Biemond, W. P. M. H. Heemels, and N. van de Wouw, "Definitions of incremental stability for hybrid systems," in IEEE Conference on Decision and Control, Osaka: Japan, 2015.

[20] R. Goebel, R. G. Sanfelice, and A. R. Teel, Hybrid dynamical systems. Princeton University Press, Princeton, U.S.A., 2012.

[21] R. I. Leine and N. van de Wouw, Stability and convergence of mechanical systems with unilateral constraints, ser. Lecture Notes in Applied and Computational Mechanics, vol. 36. Springer-Verlag, Berlin Heidelberg New-York, 2008.

[22] _ - "Uniform convergence of monotone measure differential inclusions: with application to the control of mechanical systems with unilateral constraints," International Journal of Bifurcation and Chaos, vol. 18 , no. 5, pp. 1435-1457, 2008.

[23] J. J. B. Biemond, N. van de Wouw, W. P. M. H. Heemels, and H. Nijmeijer, "Tracking control for hybrid systems with state-triggered jumps," IEEE Transactions on Automatic Control, vol. 58, no. 4, pp. 876-890, 2013.

[24] F. Forni, A. R. Teel, and L. Zaccarian, "Follow the bouncing ball: global results on tracking and state estimation with impacts," IEEE Transactions on Automatic Control, vol. 58, pp. 1470-1485, 2013.

[25] W. Lohmiller and J.-J. Slotine, "On contraction analysis for non-linear systems," Automatica, vol. 34, no. 6, pp. 683-696, 1998.

[26] W. P. M. H. Heemels, K. H. Johansson, and P. Tabuada, "An introduction to event-triggered and self-triggered control," in IEEE Conference on Decision and Control, Maui, U.S.A., 2012, pp. 3270-3285.

[27] F. H. Clarke, Optimization and nonsmooth analysis. Classics in Applied Mathematics vol. 5, SIAM, Philadelphia, U.S.A., 1990.

[28] A. R. Teel and L. Praly, "A smooth Lyapunov function from a class- $\mathcal{K} \mathcal{L}$ estimate involving two positive semidefinite functions," ESAIM: Control, Optimisation and Calculus of Variations, vol. 5, no. 1, pp. 313-367, 2000.

[29] G. A. Leonov, I. M. Burkin, and A. I. Shepeljavyi, Frequency methods in oscillation theory. Kluwer Academic Publishers Dordrecht-BostonLondon, 1996.

[30] H. K. Khalil, Nonlinear systems, 3rd ed. New Jersey, U.S.A.: PrenticeHall, Englewood Cliffs, 2002.

[31] R. G. Sanfelice, J. J. B. Biemond, N. van de Wouw, and W. P. M. H. Heemels, "An embedding approach for the design of state-feedback tracking controllers for references with jumps," International Journal of Robust and Nonlinear Control, 2013.

[32] J. J. B. Biemond, W. P. M. H. Heemels, R. G. Sanfelice, and N. van de Wouw, "Distance function design and Lyapunov techniques for the stability of hybrid trajectories," Automatica, vol. 73, pp. 38-46, 2016.

[33] K. E. Årzén, "A simple event-based PID controller," in Proceeding. of the 14th IFAC World Congress, Beijing, China, vol. 18, 1999, pp. $423-428$

[34] J. Lofberg, "YALMIP: A toolbox for modeling and optimization in MATLAB," in IEEE International Symposium on Computer Aided Control Systems Design, 2005, pp. 284-289.

[35] D. Liberzon, D. Nešić, and A. Teel, "Lyapunov-based small-gain theorems for hybrid systems," IEEE Transactions on Automatic Control, to appear.

[36] R. Postoyan, P. Tabuada, D. Nešić, and A. Anta, "A framework for the event-triggered stabilization of nonlinear systems," IEEE Transactions on Automatic Control, vol. 60, no. 4, pp. 982-996, 2015.

[37] J. J. B. Biemond, R. Postoyan, W. P. M. H. Heemels and $\mathrm{N}$. van de Wouw, "Technical report on incremental stability of hybrid systems," KU Leuven, Tech. Rep., 2016, https://people.cs.kuleuven.be/ benjamin.biemond/pdfs/hybridIS.pdf.

\section{APPENDIX A}

PROOFS

Proof of Proposition 1: First, it is shown that for any maximal solution $\phi, \sup _{j} \operatorname{dom} \phi$ is either 0 or $\infty$. Then, it is shown that $\sup _{j} \operatorname{dom} \phi=\infty$ implies $\sup _{t} \operatorname{dom} \phi=0$. With these two results, the proposition is proved.
Assume, for the sake of contradiction, that there exists a maximal solution $\phi$ to (1) for which $\sup _{j} \operatorname{dom} \phi$ is finite and nonzero. Then there exists a hybrid time $(\tilde{t}, \tilde{j}) \in \operatorname{dom} \phi$ for which $(\tilde{t}, \tilde{j}+1) \in \operatorname{dom} \phi$. Consider the two maximal solutions $\phi_{1}, \phi_{2}$ to (1) defined as $\phi_{1}(t, j)=\phi(t+\tilde{t}, j+\tilde{j})$ for $(t, j) \in$ $\operatorname{dom} \phi_{1}:=\left\{(t, j) \in \mathbb{R}_{\geq 0} \times \mathbb{N}_{0}:(t+\tilde{t}, j+\tilde{j}) \in \operatorname{dom} \phi\right\}$ and $\phi_{2}(t, j)=\phi(t+\tilde{t}, j+\tilde{j}+1)$ for $(t, j) \in \operatorname{dom} \phi_{2}:=$ $\left\{(t, j) \in \mathbb{R}_{\geq 0} \times \mathbb{N}_{0}:(t+\tilde{t}, j+\tilde{j}+1) \in \operatorname{dom} \phi\right\}$. Then $\sup _{j} \operatorname{dom} \phi_{1}=1+\sup _{j} \operatorname{dom} \phi_{2}$. As all maximal solutions are complete (since system (1) is assumed to be $\delta$-UIS), for all $\Theta$ there exists $\left(t_{1}, j_{1}\right) \in \operatorname{dom} \phi_{1}$, with $t_{1}+j_{1}>\Theta$ and $j_{1}=\sup _{j} \operatorname{dom} \phi_{1}$. We then observe that $\left(t^{\prime}, j_{1}\right) \notin \operatorname{dom} \phi_{2}$ for all $t^{\prime} \in \mathbb{R}_{>0}$. Consequently, a contradiction with item (ii) of Definition 3 is attained, such that system (1) cannot be $\delta$ UIS. We have proved that $\sup _{j} \operatorname{dom} \phi$ is either 0 or $\infty$ for all maximal solutions $\phi$ to system (1).

Now, for the sake of contradiction, assume there exists a solution $\phi$ to (1) with $\sup _{j} \operatorname{dom} \phi=\infty$ and $\sup _{t} \operatorname{dom} \phi \neq 0$. Then we can select times $\left(\tilde{t}_{1}^{j}, \tilde{j}\right) \in \operatorname{dom} \phi$ and $\left(\tilde{t}_{2}^{j}, \tilde{j}\right) \in \operatorname{dom} \phi$ with $\tilde{t}_{2}^{j}<\tilde{t}_{1}^{j}$. Consider the maximal solutions $\phi_{3}(t, j)=\phi(t+$ $\left.\tilde{t}_{1}^{j}, j+\tilde{j}\right)$ for $(t, j) \in \operatorname{dom} \phi_{3}:=\left\{(t, j) \in \mathbb{R}_{\geq 0} \times \mathbb{N}_{0}:(t+\right.$ $\left.\left.\tilde{t}_{1}^{j}, j+\tilde{j}\right) \in \operatorname{dom} \phi\right\}$ and $\phi_{4}(t, j)=\phi\left(t+\tilde{t}_{2}^{j}, j+\tilde{j}\right)$ for $(t, j) \in$ $\operatorname{dom} \phi_{4}:=\left\{(t, j) \in \mathbb{R}_{>0} \times \mathbb{N}_{0}:\left(t+\tilde{t}_{2}^{j}, j+\tilde{j}\right) \in \operatorname{dom} \phi\right\}$. As $\sup _{j} \operatorname{dom} \phi=\sup _{j} \operatorname{dom} \phi_{3}=\infty$, for all $\Theta$, there exists $(t, j) \in \operatorname{dom} \phi_{3}$, with $t+j>\Theta$, such that $(t, j) \in \operatorname{dom} \phi_{3}$ and $(t, j+1) \in \operatorname{dom} \phi_{3}$. However, $\left(t^{\prime}, j+1\right) \notin \operatorname{dom} \phi_{4}$ for $\left|t^{\prime}-t\right|<$ $\left|\tilde{t}_{1}^{j}-\tilde{t}_{2}^{j}\right|$. Hence, if $\varepsilon>0$ is selected smaller than $\left|\tilde{t}_{1}^{j}-\tilde{t}_{2}^{j}\right|$, then item (ii) of Definition 3 cannot hold. A contradiction is attained, such that we have proved that for all solutions $\phi$ to (1) $\sup _{j} \operatorname{dom} \phi=\infty$ implies $\sup _{t} \operatorname{dom} \phi=0$.

As a consequence, for all solutions $\phi$ to (1), either $\sup _{j} \operatorname{dom} \phi=0$ or $\sup _{t} \operatorname{dom} \phi=0$ holds and, since all solutions are complete, these cases imply $\sup _{t} \operatorname{dom} \phi=\infty$ or $\sup _{j} \operatorname{dom} \phi=\infty$, respectively. If there exist one maximal solution $\phi_{1}$ for which $\sup _{j}$ dom $\phi_{1}=0$ and a second maximal solution $\phi_{2}$ for which $\sup _{t} \operatorname{dom} \phi_{2}=0$, then item (ii) of Definition 3 cannot hold. Therefore, either $\sup _{j} \operatorname{dom} \phi=0$ for all maximal solutions $\phi$, or $\sup _{t} \operatorname{dom} \phi=0$ for all maximal solutions $\phi$.

Proof of Lemma 1: In order to prove item (i) of Lemma 1 , we define the sequences $\left\{t_{j}\right\}_{j \in \mathcal{I}}$ and $\left\{t_{j}^{\prime}\right\}_{j \in \mathcal{I}^{\prime}}$ such that $^{6} \operatorname{dom} \phi_{1}=\bigcup_{j \in \mathcal{I}}\left[t_{j}, t_{j+1}\right] \times\{j\}$ and $\operatorname{dom} \phi_{2}=$ $\bigcup_{j \in \mathcal{I}^{\prime}}\left[t_{j}^{\prime}, t_{j+1}^{\prime}\right] \times\{j\}$, with $\mathcal{I}=\left\{0,1, \ldots, \sup _{j} \operatorname{dom} \phi_{1}\right\}$ and $\mathcal{I}^{\prime}=\left\{0,1, \ldots, \sup _{j} \operatorname{dom} \phi_{2}\right\}$.

We define $\bar{\phi}$ with the following reasoning. Let $\bar{t}_{0}=0$ and $\bar{t}_{1}=\min \left(t_{1}, t_{1}^{\prime}\right)$, such that no jumps have occurred yet for the solutions $\phi_{1}$ and $\phi_{2}$ on $\left[\bar{t}_{0}, \bar{t}_{1}\right)$. We define $\bar{\phi}(t, j)=$ $\left(\phi_{1}(t, 0), \phi_{2}(t, 0)\right)$ for $(t, j) \in\left[0, \bar{t}_{1}\right] \times\{0\}$. For $j=1$, we consider two cases. If $\bar{t}_{1}=t_{1}$, then a jump of $\phi_{1}$ occurs at $\left(\bar{t}_{1}, 0\right)$, and we define $\bar{\phi}(t, 1)=\left(\phi_{1}(t, 1), \phi_{2}(t, 0)\right)$ for $(t, 1) \in\left[\bar{t}_{1}, \bar{t}_{2}\right]$, where we set $\bar{t}_{2}=\min \left(t_{2}, t_{1}^{\prime}\right)$. In the opposite case $\bar{t}_{1}=t_{1}^{\prime}<t_{1}$, we take $\bar{\phi}(t, j)=\left(\phi_{1}(t, 0), \phi_{2}(t, 1)\right)$ for $(t, j) \in\left[\bar{t}_{1}, \bar{t}_{2}\right] \times\{1\}$ and define $\bar{t}_{2}=\min \left(t_{1}, t_{2}^{\prime}\right)$. We note that in both cases, $\bar{\phi}$ is a solution to (3) in the time domain $\left[\bar{t}_{0}, \bar{t}_{1}\right] \times\{0\} \cup\left[\bar{t}_{1}, \bar{t}_{2}\right] \times\{1\}$. In order to repeat this argument

\footnotetext{
${ }^{6}$ The last continuous-time interval of dom $\phi_{1}$ will be open on the right if $\tilde{\jmath}=\sup _{j} \operatorname{dom} \phi_{1}$ is finite and $\sup _{t} \operatorname{dom} \phi_{1}=t_{\tilde{\jmath}+1}=\infty$
} 
and extend the description of $\bar{\phi}$ iteratively, we require to know, for the last known time instant $(t, j) \in \operatorname{dom} \bar{\phi}$, how many jumps of $\phi_{1}$ and $\phi_{2}$ have occurred. We use counters for this purpose and denote them by $j_{1}(j)$ and $j_{2}(j)$, for $j \in\left\{0,1, \ldots, \sup _{j} \operatorname{dom} \bar{\phi}\right\}$, respectively. In this manner, we obtain the iterative definition $\left(\bar{t}_{0}, j_{1}(0), j_{2}(0)\right)=(0,0,0)$ and, for $k \in \overline{\mathcal{I}}:=\left\{0,1, \ldots, \sup _{j} \operatorname{dom} \phi_{1}+\sup _{j} \operatorname{dom} \phi_{2}-1\right\}$,

$$
\left(\bar{t}_{k+1}, j_{1}(k+1), j_{2}(k+1)\right)=\left\{\begin{array}{c}
\left(t_{j_{1}(k)+1}, j_{1}(k)+1, j_{2}(k)\right), \\
\text { if } t_{j_{1}(k)+1} \leq t_{j_{2}(k)+1}^{\prime}, \\
\left(t_{j_{2}(k)+1}^{\prime}, j_{1}(k), j_{2}(k)+1\right), \\
\text { if } t_{j_{1}(k)+1}>t_{j_{2}(k)+1}^{\prime} .
\end{array}\right.
$$

The time instants $\left\{\bar{t}_{k}\right\}_{k \in \overline{\mathcal{I}}}$ correspond to jumps of either $\phi_{1}$ or $\phi_{2}$. In the construction above, we only increase the counter $j_{1}(k)$ with 1 if the jump time $\left(\bar{t}_{k+1}, k\right)$ corresponds to a jump of $\phi_{1}$, otherwise, we only increase $j_{2}(k)$. Since both $\phi_{1}$ and $\phi_{2}$ are $t$-complete, the sequence above is defined for any $k$ in $\overline{\mathcal{I}}$.

In view of (18), $\bar{t}_{k+1} \leq t_{j_{1}(k)+1}$. Since $\bar{t}_{0}=t_{0}=0$ and $j_{1}(k+1)=j_{1}(k)+1$ occurs simultaneously with $\bar{t}_{k+1}=$ $t_{j_{1}(k)+1}$, we deduce that $\bar{t}_{k} \geq t_{j_{1}(k)}$ for all $k \in \overline{\mathcal{I}}$. Hence, we find $\left[\bar{t}_{k}, \bar{t}_{k+1}\right] \times\left\{j_{1}(k)\right\} \subset \operatorname{dom} \phi_{1}$ and analogously, we derive $\left[\bar{t}_{k}, \bar{t}_{k+1}\right] \times\left\{j_{2}(k)\right\} \subset \operatorname{dom} \phi_{2}$. Consequently, we define the hybrid arc $\bar{\phi}$ as

$$
\bar{\phi}(t, \bar{j})=\left(\phi_{1}\left(t, j_{1}(\bar{j})\right), \phi_{2}\left(t, j_{2}(\bar{j})\right)\right),
$$

for all $(t, \bar{j}) \in \operatorname{dom} \phi:=\bigcup_{j \in \overline{\mathcal{I}}}\left[\bar{t}_{j}, \bar{t}_{j+1}\right] \times\{j\}$. We observe that (18) yields the sequence of continuous times $\left\{\bar{t}_{j}\right\}_{j \in \overline{\mathcal{I}}}$ obtained by sorting $\left\{t_{j}\right\}_{j \in \mathcal{I}} \cup\left\{t_{j}^{\prime}\right\}_{j \in \mathcal{I}^{\prime}}$. Hence $\operatorname{dom} \bar{\phi}=$ $\bigcup_{j \in \overline{\mathcal{I}}}\left[\bar{t}_{j}, \bar{t}_{j+1}\right] \times\{j\}$ is a hybrid time domain (see Section II).

We now show that (19) is a solution of (3) by checking the properties given in Section $\mathrm{II}^{7}$. Clearly, $\bar{\phi}(0,0) \in$ $C_{\mathrm{f}} \cup D_{\mathrm{f}}$ as $\phi_{1}(0,0), \phi_{2}(0,0) \in C \cup D$. In addition, for those $j$ where $\bar{t}_{j+1}>\bar{t}_{j}$, we observe that $\frac{d}{d t} \bar{\phi}(t, j)=$ $\left(\frac{d}{d t} \phi_{1}\left(t, j_{1}(j)\right), \frac{d}{d t} \phi_{2}\left(t, j_{2}(j)\right)\right)$ holds for almost all $t \in$ $\left[\bar{t}_{j}, \bar{t}_{j+1}\right]$. Hence, $\frac{d}{d t} \bar{\phi}(t, j) \in F_{\mathrm{f}}(\bar{\phi}(t, j))$ holds for almost all $t \in\left[\bar{t}_{j}, \bar{t}_{j+1}\right]$ by construction of $F_{\mathrm{f}}$. Furthermore, if $\bar{t}_{j+1}=t_{j_{1}(j)+1}<\infty$, we observe that, firstly, $\bar{\phi}\left(\bar{t}_{j+1}, j\right) \in D_{\mathrm{f}}$ as $\bar{\phi}\left(\bar{t}_{j+1}, j\right)=\left(\phi_{1}\left(t_{j_{1}(j)+1}, j_{1}(j)\right), \phi_{2}\left(t_{j_{1}(j)+1}, j_{2}(j)\right)\right) \in$ $D \times(C \cup D)$ and, secondly, that

$$
\begin{aligned}
\bar{\phi}\left(\bar{t}_{j+1}, j+1\right) & =\left(\phi_{1}\left(\bar{t}_{j+1}, j_{1}(j+1)\right), \phi_{2}\left(\bar{t}_{j+1}, j_{2}(j+1)\right)\right) \\
& =\left(\phi_{1}\left(\bar{t}_{j+1}, j_{1}(j)+1\right), \phi_{2}\left(\bar{t}_{j+1}, j_{2}(j)\right)\right),
\end{aligned}
$$

such that we find $\bar{\phi}\left(\bar{t}_{j+1}, j+1\right) \quad \in$ $\left(G\left(\phi_{1}\left(\bar{t}_{j+1}, j_{1}(j)\right)\right),\left\{\phi_{2}\left(\bar{t}_{j+1}, j_{2}(j)\right)\right\}\right) \subseteq G_{\mathrm{f}}\left(\bar{\phi}_{(}\left(\bar{t}_{j+1}, j\right)\right)$. Analogously, we can prove that if $\bar{t}_{j+1}=t_{j_{2}(j)+1}^{\prime}<\infty$, then $\bar{\phi}\left(\bar{t}_{j+1}, j\right) \in D_{\mathrm{f}}$ and $\bar{\phi}\left(\bar{t}_{j+1}, j+1\right) \in$ $\left(\left\{\phi_{1}\left(\bar{t}_{j+1}, j_{1}(j)\right)\right\}, G\left(\phi_{2}\left(\bar{t}_{j+1}, j_{2}(j)\right)\right)\right) \subseteq G_{\mathrm{f}}\left(\bar{\phi}\left(\bar{t}_{j+1}, j\right)\right)$ Hence, $\bar{\phi}\left(\bar{t}_{j+1}, j+1\right) \in G_{\mathrm{f}}\left(\bar{\phi}\left(\bar{t}_{j+1}, j\right)\right)$ and $\bar{\phi}\left(\bar{t}_{j+1}, j\right) \in D_{\mathrm{f}}$ holds for all $j \geq 0$ with $j+1 \in \overline{\mathcal{I}}$. Consequently, $\bar{\phi}$ is a solution to (3).

To conclude the proof of item (i) of Lemma 1, given $\phi_{1}, \phi_{2}$, we select $\bar{\phi}, j_{1}, j_{2}$ as above and we observe that for every $(t, j) \in \operatorname{dom} \phi_{1}$, we can select a $\bar{j}$ such that $j=j_{1}(\bar{j})$ and

\footnotetext{
${ }^{7}$ Note that system (3) satisfies the hybrid basic conditions.
}

$\left(t, j^{\prime}\right)=\left(t, j_{2}(\bar{j})\right) \in \operatorname{dom} \phi_{2}$. Hence, the statement (6) is attained from the observation that $\bar{j}=j_{1}(\bar{j})+j_{2}(\bar{j})$, which holds as $j_{1}(0)=j_{2}(0)=0$ and $j_{1}(\bar{j}+1)+j_{2}(\bar{j}+1)=$ $j_{1}(\bar{j})+j_{2}(\bar{j})+1$ for all $\bar{j}$, in view of $(18)$.

To prove item (ii) of Lemma 1 , consider a solution $\bar{\phi}$ and introduce the sequence of jump times $\left\{\bar{t}_{j}\right\}_{\overline{\mathcal{I}}}$, with $\overline{\mathcal{I}}=$ $\left\{0,1, \ldots, \sup _{j} \operatorname{dom} \bar{\phi}\right\}$. For every $\bar{j} \in \overline{\mathcal{I}}$, let $\jmath_{1}(\bar{j})$ denote the cardinality of the set

$\left\{j \in\{1, \ldots \bar{j}\}: \bar{\phi}\left(\bar{t}_{j}, j\right) \in\left(G\left(\bar{\phi}_{1}\left(\bar{t}_{j}, j-1\right)\right),\left\{\bar{\phi}_{2}\left(\bar{t}_{j}, j-1\right)\right\}\right)\right\}$

where $\bar{\phi}=\left(\bar{\phi}_{1}, \bar{\phi}_{2}\right)$ and let $\jmath_{2}(\bar{j})$ denote the cardinality of the set

$\left\{j \in\{1, \ldots \bar{j}\}: \bar{\phi}\left(\bar{t}_{j}, j\right) \in\left(\left\{\bar{\phi}_{1}\left(\bar{t}_{j}, j-1\right)\right\}, G\left(\bar{\phi}_{2}\left(\bar{t}_{j}, j-1\right)\right)\right)\right\}$.

As $G(x) \neq\{x\}$ for all $x \in D$ since all maximal solution to (1) are $t$-complete by assumption, we observe that for any $j \in$ $\{1, \ldots \bar{j}\}$ either $\bar{\phi}\left(\bar{t}_{j}, j\right) \in\left(G\left(\bar{\phi}_{1}\left(\bar{t}_{j}, j-1\right)\right),\left\{\bar{\phi}_{2}\left(\bar{t}_{j}, j-1\right)\right\}\right)$ or $\bar{\phi}\left(\bar{t}_{j}, j\right) \in\left(\left\{\bar{\phi}_{1}\left(\bar{t}_{j}, j-1\right)\right\}, G\left(\bar{\phi}_{2}\left(\bar{t}_{j}, j-1\right)\right)\right)$ holds, but not both. Hence, with (21) and (22) we observe that $\bar{j}=$ $\jmath_{1}(\bar{j})+\jmath_{2}(\bar{j})$.

The hybrid time domain $\operatorname{dom} \bar{\phi}$ and the functions $\jmath_{1}, \jmath_{2}$ defined above allows to define the hybrid time domains

$$
\begin{aligned}
\operatorname{dom} \phi_{1} & =\left\{(t, j):(t, j)=\left(t, \jmath_{1}(\bar{j})\right),(t, \bar{j}) \in \operatorname{dom} \bar{\phi}\right\} \\
\operatorname{dom} \phi_{2} & =\left\{(t, j):(t, j)=\left(t, \jmath_{2}(\bar{j})\right),(t, \bar{j}) \in \operatorname{dom} \bar{\phi}\right\}
\end{aligned}
$$

and using the hybrid arc $\bar{\phi}$, we define the hybrid arcs

$$
\phi_{1}(t, j)=\bar{\phi}_{1}\left(t, \min \left\{\bar{j}: \jmath_{1}(\bar{j})=j,(t, \bar{j}) \in \operatorname{dom} \bar{\phi}\right\}\right)
$$

for $(t, j) \in \operatorname{dom} \phi_{1}$

$$
\phi_{2}(t, j)=\bar{\phi}_{2}\left(t, \min \left\{\bar{j}: \jmath_{2}(\bar{j})=j,(t, \bar{j}) \in \operatorname{dom} \bar{\phi}\right\}\right),
$$

for $(t, j) \in \operatorname{dom} \phi_{2}$. Observe that $\operatorname{dom} \phi_{1}$ and $\operatorname{dom} \phi_{2}$ are hybrid time domains and $\phi_{1}, \phi_{2}$ are solutions to (1). For any $(t, j) \in \operatorname{dom} \phi_{1}$, we can select some $\bar{j} \in \overline{\mathcal{I}}$ such that $j=$ $\jmath_{1}(\bar{j})$. Taking $j^{\prime}=\jmath_{2}(\bar{j})$, item (ii) of Lemma 1 follows from $\bar{j}=\jmath_{1}(\bar{j})+\jmath_{2}(\bar{j})$ obtained above.

Proof of Lemma 2: To prove the only if-statement, we suppose, for the sake of contradiction, that there exists a maximal solution $\bar{\phi}$ to (3) such that $\sup _{t} \operatorname{dom} \bar{\phi}=T<\infty$ while all maximal solutions $\phi$ to (1) satisfy $\sup _{t} \operatorname{dom} \phi=\infty$. Let $\phi_{1}, \phi_{2}$ be maximal solutions to (1) as in item (ii) of Lemma 1. Introducing $J=\sup _{j} \operatorname{dom} \bar{\phi}$, we distinguish the case $(T, J) \in \operatorname{dom} \bar{\phi}$ from the case $(T, J) \notin \operatorname{dom} \bar{\phi}$.

If $(T, J) \in \operatorname{dom} \bar{\phi}$, then we consider $j, j^{\prime}$ with $J=$ $j+j^{\prime}$, such that $\bar{\phi}(T, J)=\left(\phi_{1}(T, j), \phi_{2}\left(T, j^{\prime}\right)\right)$, in view of Lemma 1, item (ii). As the solutions $\phi_{1}, \phi_{2}$ can be continued from time $(T, j)$ and $\left(T, j^{\prime}\right)$, respectively, we conclude that either $(T, j+1) \in \operatorname{dom} \phi_{1}$, or $\left(T, j^{\prime}+1\right) \in \operatorname{dom} \phi_{2}$, or there exist $\bar{s}>0$ such that for $s \in[0, \bar{s}],(T+s, j) \in \operatorname{dom} \phi_{1}$ and $(T+s, j) \in \operatorname{dom} \phi_{2}$. In the first and second option, we conclude that $\bar{\phi}(T, j) \in D_{\mathrm{f}}$, and $\bar{\phi}$ is not maximal, yielding a contradiction. Given the third option, we observe that $\bar{\phi}$ can be extended with $\bar{\phi}(t, J)=\left(\phi_{1}(t, j), \phi_{2}\left(t, j^{\prime}\right)\right.$ for $t \in[T, T+s]$, contradicting $\sup _{t} \operatorname{dom} \bar{\phi}=T$. Hence, a contradiction is found for every option where $(T, J) \in \operatorname{dom} \bar{\phi}$ holds. 
If $(T, J) \notin \operatorname{dom} \bar{\phi}$ we can select a sequence of hybrid times $\left(T_{i}, J_{i}\right) \in \operatorname{dom} \bar{\phi}$, with $\lim _{i \rightarrow \infty} T_{i}=T$ and $\lim _{i \rightarrow \infty} J_{i}=J$. Applying item (ii) of Lemma 1 for each of these hybrid times, we can further select sequences $\left\{j_{i}\right\}_{i \in \mathbb{N}},\left\{j_{i}^{\prime}\right\}_{i \in \mathbb{N}}$, such that $j_{i}+j_{i}^{\prime}=J_{i},\left(T_{i}, j_{i}\right) \in \operatorname{dom} \phi_{1}$ and $\left(T_{i}, j_{i}^{\prime}\right) \in \operatorname{dom} \phi_{2}$.

- If $J=\infty$, either $\lim _{i \rightarrow \infty} j_{i}=\infty$ or $\lim _{i \rightarrow \infty} j_{i}^{\prime}=$ $\infty$. The first case $\lim _{i \rightarrow \infty} j_{i}=\infty$ implies that $\sup _{t} \operatorname{dom} \phi_{1} \leq T$, since otherwise, there exists some $(\tau, \kappa) \in \operatorname{dom} \phi_{1}$, with $\tau>T$ and some $i$ for which $j_{i}>\kappa$ holds, yielding a contradiction as two hybrid times $\left(T_{i}, j_{i}\right)$ and $(\tau, \kappa)$ can never be contained in the same hybrid time domain dom $\phi_{1}$ as $T_{i} \leq T<\tau$ and $j_{i}>\kappa$. Hence, $\lim _{i \rightarrow \infty} j_{i}=\infty$ implies $\sup _{t} \operatorname{dom} \phi_{1} \leq T$ and similarly, we observe that $\lim _{i \rightarrow \infty} j_{i}^{\prime}=\infty$ implies $\sup _{t} \operatorname{dom} \phi_{2} \leq T$. In both cases, a contradiction is attained.

- If $J<\infty$, we observe that $\operatorname{dom} \bar{\phi} \cap\left(\mathbb{R}_{>0} \times\{J\}\right)=$ $[\tilde{T}, T) \times\{J\}$ for some $\tilde{T} \in[0, T)$, and there does not exists an absolutely continuous function $z:[a, b] \rightarrow C$ satisfying $\dot{z}(t)=F_{\mathrm{f}}(z(t))$ for almost all $t \in[a, b]$, with $[\tilde{T}, T) \subset[a, b]$ and $z(t)=\bar{\phi}(t, J)$, cf. [20, Proposition 2.10]. By (A2) of the hybrid basic conditions, $F$ is convex-valued, outer semicontinuous and locally bounded relative to $C$, such that the same properties hold for $F_{\mathrm{f}}$ and $C_{\mathrm{f}}$ and we can infer $\lim _{i \rightarrow \infty}\left\|\bar{\phi}\left(T_{i}, J_{i}\right)\right\|=\infty$, where we exploited that $C_{\mathrm{f}}$ is a closed set. However, in that case either $\lim \left\|\phi_{1}\left(T_{i}, j_{i}\right)\right\|=\infty$ and $\sup _{t} \operatorname{dom} \phi_{1}=T$, or $\lim _{i \rightarrow \infty}\left\|\phi_{2}\left(T_{i}, j_{i}^{\prime}\right)\right\|=\infty$ and $\sup _{t} \operatorname{dom} \phi_{2}=T$, obtaining a contradiction.

In all cases, a contradiction is found, proving only if.

To prove the if-statement, we suppose, for the sake of contradiction, that there exist a maximal solution $\phi_{1}$ to (1), where $\sup _{t} \operatorname{dom} \phi_{1}=T<\infty$, while all maximal solutions $\bar{\phi}$ to (3) satisfy $\sup _{t} \operatorname{dom} \bar{\phi}=\infty$. We select $\phi_{2}=\phi_{1}$ and construct $\bar{\phi}$ such that (6) holds. Let $J=\sup _{j} \operatorname{dom} \phi_{1}$ and introduce $\left\{t_{j}\right\}_{j \in\{0,1, \ldots J+1\}}$ such that $\operatorname{dom} \phi_{1}=\bigcup_{j \in\{0,1, \ldots J\}}\left[t_{j}, t_{j+1}\right] \times$ $\{j\}$. We define the set $\operatorname{dom} \bar{\phi}=\left(\bigcup_{j \in\{0,1, \ldots J\}}\left[t_{j}, t_{j+1}\right] \times\right.$ $\{2 j\}) \cup\left(\bigcup_{j \in\{0,1, \ldots J\}}\left\{t_{j+1}\right\} \times\{2 j+1\}\right)$ and note that this is indeed a hybrid time domain. On this domain, we define

$$
\bar{\phi}(t, \bar{j})=\left\{\begin{array}{c}
\left(\phi_{1}\left(t, \frac{1}{2} \bar{j}\right),\left(\phi_{1}\left(t, \frac{1}{2} \bar{j}\right)\right)\right), \\
\text { for } \bar{j} \in\{0,2, \ldots 2 J\} \\
\left(\phi_{1}\left(t, \frac{1}{2}(\bar{j}+1)\right),\left(\phi_{1}\left(t, \frac{1}{2}(\bar{j}-1)\right)\right)\right), \\
\text { for } \bar{j} \in\{1,3, \ldots 2 J-1\}
\end{array}\right.
$$

that is a solution to (3), which can be extended as all maximal solutions to (3) are $t$-complete. Denoting this extension as $\bar{\phi}^{e}$ and applying item (ii) of Lemma 1, we find solutions $\phi_{1}^{e}, \phi_{2}^{e}$ that are extensions of $\phi_{1}$ since $\sup _{t} \operatorname{dom} \phi_{1}^{e}=\sup _{t} \operatorname{dom} \phi_{2}^{e}=$ $\sup _{t} \operatorname{dom} \bar{\phi}=\infty$, contradicting that $\phi_{1}$ is maximal. A contradiction is found, proving the if-statement.

Proof of Theorem 1: We first study the implication (i) $\Rightarrow$ (ii). Assuming system (3) is $\delta$ - $\mathrm{U}_{\mathrm{t}} \mathrm{GAS}$, by Definition 1 , all maximal solutions to (3) are $t$-complete. Consequently, from Lemma 2, we infer all maximal solutions to (1) are $t$-complete.

We prove that the system is $\delta$-tFUIS as stated in Definition 5 by first showing that item (i) of Definition 4 holds for $t^{\prime}=t$. Given any $\varepsilon>0$, select $s>0$ as in item (i) of
Definition 1, which can be done given item (i) of Theorem 1. Consider two maximal solutions $\phi_{1}$ and $\phi_{2}$ to (1) such that $\delta\left(\phi_{1}(0,0), \phi_{2}(0,0)\right)<s$. Introducing the solution $\bar{\phi}$ to system (3) given by item (i) of Lemma 1, we observe that for any $(t, j) \in \operatorname{dom} \phi_{1}$, there exists $\left(t, j^{\prime}\right) \in \operatorname{dom} \phi_{2}$ such that $\bar{\phi}\left(t, j+j^{\prime}\right)=\left(\phi_{1}(t, j), \phi_{2}\left(t, j^{\prime}\right)\right)$. From item (i) of Definition 1 , we deduce that $\delta\left(\phi_{1}(t, j), \phi_{2}\left(t, j^{\prime}\right)\right)=\delta\left(\bar{\phi}\left(t, j+j^{\prime}\right)\right)<\varepsilon$ (since $\left.\delta\left(\phi_{1}(0,0), \phi_{2}(0,0)\right)=\delta(\bar{\phi}(0,0))<s\right)$. Hence, item (i) of Definition 4 holds with $t^{\prime}=t$, as imposed by Definition 5

To infer item (ii) of Definition 4, consider any pair $\varepsilon, r$ with $\varepsilon>0$ and $r>0$. Let $T>0$ satisfy item (ii) of Definition 1 . We consider two maximal solutions $\phi_{1}$ and $\phi_{2}$ to (1) such that $\delta\left(\phi_{1}(0,0), \phi_{2}(0,0)\right)<r$ and we construct $\bar{\phi}$ using item (i) of Lemma 1 . For any $(t, j) \in \operatorname{dom} \phi_{1}$ with $t \geq T$, there exists $\left(t, j^{\prime}\right) \in \operatorname{dom} \phi_{2}$ such that $\delta\left(\phi_{1}(t, j), \phi_{2}\left(t, j^{\prime}\right)\right)=\delta(\bar{\phi}(t, j+$ $\left.\left.j^{\prime}\right)\right)$. Since $t \geq T, \delta\left(\phi_{1}(t, j), \phi_{2}\left(t, j^{\prime}\right)\right)=\delta\left(\bar{\phi}\left(t, j+j^{\prime}\right)\right)<\varepsilon$ follows from item (ii) of Definition 1. Hence, item (ii) of Definition 4 holds when $t^{\prime}=t$ is selected.

We now concentrate on the converse implication and assume that item (ii) of Theorem 1 holds. Consequently, as (1) is $\delta$ tFUIS, all its maximal solutions $\phi$ are $t$-complete, such that we deduce from Lemma 2 that any maximal solution $\bar{\phi}$ to (3) is $t$-complete as well.

To prove item (i) of Definition 1, we assume for the sake of contradiction that (3) is not stable with respect to $\delta$, i.e. there exists $\varepsilon>0$ such that, for all $s>0$ there exists a solution $\bar{\phi}$ to (3) and time instant $(t, \bar{j}) \in \operatorname{dom} \bar{\phi}$ such that

$$
\delta(\bar{\phi}(0,0))<s \text { and } \delta(\bar{\phi}(t, \bar{j})) \geq \varepsilon .
$$

Given this solution $\bar{\phi}$ and hybrid time $(t, \bar{j}) \in \operatorname{dom} \bar{\phi}$, let $\phi_{1}, \phi_{2},(t, j) \in \operatorname{dom} \phi_{1},\left(t, j^{\prime}\right) \in \operatorname{dom} \phi_{2}$ be selected as in item (ii) of Lemma 1. Substituting (6) in (28) and using $\bar{\phi}(0,0)=\left(\phi_{1}(0,0), \phi_{2}(0,0)\right)$ then yields

$$
\delta\left(\phi_{1}(0,0), \phi_{2}(0,0)\right)<s \text { and } \delta\left(\phi_{1}(t, j), \phi_{2}\left(t, j^{\prime}\right)\right) \geq \varepsilon \text {. }
$$

For the considered $\varepsilon, s$ can be chosen arbitrarily, which contradicts Definition 5 since item (i) of Definition 4 cannot hold with $t^{\prime}=t$. This contradiction proves that $\delta$-tFUIS of (1) implies stability with respect to $\delta$ of (3).

To prove item (ii) of Definition 1 we assume, for the sake of contradiction, that there exists $\varepsilon, r>0$ such that for all $T>0$, there exists a solution $\bar{\phi}$ to (3) such that

$$
\delta(\bar{\phi}(0,0))<r \text { and } \delta(\bar{\phi}(t, \bar{j})) \geq \varepsilon
$$

for some $(t, \bar{j}) \in \operatorname{dom} \bar{\phi}$ such that $t>T$. Let $\phi_{1}, \phi_{2},(t, j) \in$ $\operatorname{dom} \phi_{1},\left(t, j^{\prime}\right) \in \operatorname{dom} \phi_{2}$ with $j+j^{\prime}=\bar{j}$ be selected as in item (ii) of Lemma 1. Substituting (6) in (30) and using $\left(\bar{\phi}_{1}(0,0), \bar{\phi}_{2}(0,0)\right)=\left(\phi_{1}(0,0), \phi_{2}(0,0)\right)$ then yields $\bar{j}=$ $j+j^{\prime}, \delta\left(\phi_{1}(0,0), \phi_{2}(0,0)\right)<r$ and $\delta\left(\phi_{1}(t, j), \phi_{2}\left(t, j^{\prime}\right)\right) \geq \varepsilon$. Since for all $T>0$, we can find solutions $\phi_{1}, \phi_{2}$ such that the above inequalities hold for some $(t, j),\left(t, j^{\prime}\right)$ with $t>T$, a contradiction with item (ii) of Definition 4 is attained if $t=t^{\prime}$ is selected in that definition, as imposed by Definition 5 . Hence, system (3) is uniformly in $t$ globally attractive with respect to $\delta$. We have proved the implication (ii) $\Rightarrow$ (i), concluding the proof of the Theorem.

Proof of Proposition 2: Let $\bar{\phi}$ be a solution to (3). Let $(t, j) \in \operatorname{dom} \bar{\phi}$ and $0=t_{0} \leq t_{1} \leq \ldots \leq t_{j+1}=t$ satisfy 
$\operatorname{dom} \bar{\phi} \cap([0, t] \times\{0,1, \ldots, j\})=\bigcup_{i \in\{0,1, \ldots, j\}}\left[t_{i}, t_{i+1}\right] \times$ $\{i\}$. According to item (ii) of Proposition 2 and since $U$ is locally Lipschitz, we have, for each $i \in\{0,1, \ldots, j\}$ and for almost all $s \in\left[t_{i}, t_{i+1}\right]$ (see [27] for more details $), \frac{d}{d s} U(\bar{\phi}(s, i)) \leq-\sigma(U(\bar{\phi}(s, i)))$. By integrating both sides of this inequality, we obtain $U\left(\bar{\phi}\left(t_{i+1}, i\right)\right)-$ $U\left(\bar{\phi}\left(t_{i}, i\right)\right) \leq-\int_{t_{i}}^{t_{i+1}} \sigma(U(\bar{\phi}(s, i))) s$. Since $U$ does not increase at jumps along $\bar{\phi}$ according to item (iii) of Proposition 2 , for any $(t, j) \in \operatorname{dom} \bar{\phi}, U(\bar{\phi}(t, i)) \leq U(\bar{\phi}(0,0))-$ $\sum_{i=0}^{j} \int t_{i}^{t_{i+1}} \sigma(U(\bar{\phi}(s, i))) s$. We derive that system (3) is $\delta$ $\mathrm{U}_{\mathrm{t}} \mathrm{GpAS}$ by following the same arguments as the proof of Theorem 3.18 in [20]. From item (iv) of Proposition 2 and Lemma 2, we deduce that any maximal solution to (3) is $t$ complete, such that system (3) is $\delta$ - $\mathrm{U}_{\mathrm{t}} \mathrm{GAS}$.

Proof of Proposition 3: The desired result is attained by invoking Theorem 1. For this purpose, we first use Proposition 2 to establish that the extended system (3) for system (7) is $\delta$-U $\mathrm{U}_{\mathrm{t}} \mathrm{GAS}$. Let $U\left(q_{1}, q_{2}\right)=\delta\left(q_{1}, q_{2}\right)$ for any $q_{1}=\left(x_{1}, e_{1}\right) \in$ $\mathbb{R}^{n} \times \mathbb{R}^{n}$ and $q_{2}=\left(x_{2}, e_{2}\right) \in \mathbb{R}^{n} \times \mathbb{R}^{n}$. The function $U$ is locally Lipschitz on $\mathbb{R}^{n} \times \mathbb{R}^{n}$ since $V$ is continuously differentiable on $\mathbb{R}^{n} \times \mathbb{R}^{n}$ according to Assumption 1. Item (i) of Proposition 2 is verified with $\alpha_{1}=\alpha_{2}=\mathbb{I}$. Let $q_{1}=\left(x_{1}, e_{1}\right) \in C$ and $q_{2}=\left(x_{2}, e_{2}\right) \in C$ with $C=$ $\{(x, e):\|e\| \leq \rho\}$. We distinguish three cases. First, if $V\left(x_{1}, x_{2}\right)-a_{V}^{-1} \gamma(2 \rho)<0$, then $U\left(q_{1}, q_{2}\right)=0$ and, noting $F_{\mathrm{f}}\left(q_{1}, q_{2}\right)=\left\{\left(f\left(q_{1}\right), f\left(q_{2}\right)\right)\right\}$, we find $\left\langle\zeta,\left(f\left(q_{1}\right), f\left(q_{2}\right)\right)\right\rangle=$ $0=-\sigma\left(U\left(q_{1}, q_{2}\right)\right)$ for any $\zeta \in \partial U\left(q_{1}, q_{2}\right)$ and any positive definite function $\sigma$. When $V\left(x_{1}, x_{2}\right)-a_{V}^{-1} \gamma(2 \rho)>0$, let $\zeta \in \partial U\left(q_{1}, q_{2}\right),\left\langle\zeta,\left(f\left(q_{1}\right), f\left(q_{2}\right)\right)\right\rangle \leq-a_{V} V\left(x_{1}, x_{2}\right)+$ $\gamma\left(\left\|e_{1}-e_{2}\right\|\right)$ in view of item (ii) of Assumption 1. Since $q_{1}, q_{2} \in C, \max \left\{\left\|e_{1}\right\|,\left\|e_{2}\right\|\right\} \leq \rho$ and $\gamma\left(\left\|e_{1}-e_{2}\right\|\right) \leq$ $\gamma(2 \rho)$. Consequently, $\left\langle\zeta,\left(f\left(q_{1}\right), f\left(q_{2}\right)\right)\right\rangle \leq-a_{V} U\left(q_{1}, q_{2}\right)$. When $a_{V} V\left(x_{1}, x_{2}\right)-\gamma(2 \rho)=0$, we similarly derive that $\left\langle\zeta,\left(f\left(q_{1}\right), f\left(q_{2}\right)\right)\right\rangle \leq-a_{V} U\left(q_{1}, q_{2}\right)=0$. Therefore, according to Lemma II.1 in [35], item (ii) of Proposition 2 holds with $\sigma=a_{V} \mathbb{I}$. Item (iii) of Proposition 2 is satisfied since the $x$-variable does not change at jumps according to (7) and the expression of $U$ only depends on $x_{1}$ and $x_{2}$. Finally, any maximal solution to (7) is $t$-complete according to Theorem 5 in [36] (here $\|e\|$ plays the role of $\gamma(\|e\|)$ in [36], which is locally Lipschitz and not continuously differentiable everywhere, still the proof of Theorem 5 in [36] applies under minor changes). Hence item (iv) of Proposition 2 holds according to Lemma 2. As a consequence, the conditions of Proposition 2 are verified.

Proof of Lemma 3: To prove item (i), consider two $j$ complete solutions $\phi_{1}, \phi_{2}$ and introduce two sequences of continuous times $\left\{t_{j}^{1}\right\}_{j \in \mathbb{N}_{0}},\left\{t_{j}^{2}\right\}_{j \in \mathbb{N}_{0}}$ such that $\operatorname{dom} \phi_{i}=$ $\bigcup_{j \in \mathbb{N}_{0}}\left[t_{j}^{i}, t_{j+1}^{i}\right] \times\{j\}, i=1,2$. Let $t^{*}=\sup _{j \in \mathbb{N}_{0}} t_{j+1}^{1}+t_{j+1}^{2}$ and $\operatorname{dom} \bar{\phi}=\bigcup_{j \in \mathbb{N}_{0}}\left[t_{j}^{1}+t_{j}^{2}, t_{j+1}^{1}+t_{j+1}^{2}\right] \times\{j\}$, and observe that this set is a hybrid time domain (as defined in Section II) with $\sup _{j} \operatorname{dom} \bar{\phi}=\infty$ and $\sup _{t} \operatorname{dom} \bar{\phi}=t^{*}$.

In the following, we define continuous functions $\tau_{1}, \tau_{2}$ : $\left[0, t^{*}\right] \rightarrow \mathbb{R}_{\geq 0}$ such that the hybrid arc

$$
\bar{\phi}(t, j)=\left(\phi_{1}\left(\tau_{1}(t), j\right), \phi_{2}\left(\tau_{2}(t), j\right)\right) .
$$

is a solution to (10). For this purpose, consider the signal $v:\left[0, t^{*}\right] \rightarrow\{0,1\}^{2}$ given as

$$
v(t)= \begin{cases}(1,0), & t_{j}^{1}+t_{j}^{2} \leq t \leq t_{j+1}^{1}+t_{j}^{2} \\ (0,1), & t_{j+1}^{1}+t_{j}^{2} \leq t \leq t_{j+1}^{1}+t_{j+1}^{2}\end{cases}
$$

for $j \in \mathbb{N}_{0}$ and let $\left(\tau_{1}(t), \tau_{2}(t)\right)=\int_{0}^{t} v(s) d s$.

For $i=1,2$, we find that for each $j \in \mathbb{N}_{0},\left\{\tau_{i}(t): t \in\right.$ $\left.\left[t_{j}^{1}+t_{j}^{2}, t_{j+1}^{1}+t_{j+1}^{2}\right]\right\}=\left[t_{j}^{i}, t_{j+1}^{i}\right]$ such that we can conclude

$$
\left\{\left(\tau_{i}(t), j\right):(t, j) \in \operatorname{dom} \bar{\phi}\right\}=\operatorname{dom} \phi_{i} .
$$

We observe that the function $\bar{\phi}: \operatorname{dom} \bar{\phi} \rightarrow \mathbb{R}^{2 n}$ given by

$$
\bar{\phi}(t, j)=\left(\begin{array}{l}
\phi_{1}\left(\tau_{1}(t), j\right) \\
\phi_{2}\left(\tau_{2}(t), j\right)
\end{array}\right)
$$

is a solution to (10), as it satisfies the conditions stated in Section II: (i) $\bar{\phi}(0,0) \in C_{\mathrm{j}} \cup D_{\mathrm{j}}$, as $\tau_{1}(0)=\tau_{2}(0)=0$, (ii) for any $j \in \mathbb{N}_{0}$ and almost all $t \in\left[t_{j}^{1}+t_{j}^{2}, t_{j+1}^{1}+\right.$ $\left.t_{j}^{2}\right], \frac{d \bar{\phi}(t, j)}{d t}=\left(\frac{d \phi_{1}\left(\tau_{1}(t), j\right)}{d t}, \frac{d \phi_{2}\left(\tau_{2}(t), j\right)}{d t}\right)=\left(\frac{d \phi_{1}\left(\tau_{1}, j\right)}{d \tau_{1}}, 0\right) \in$ $\left(F\left(\phi_{1}\left(\tau_{1}, j\right)\right), 0\right) \subset F_{\mathrm{j}}(\bar{\phi}(t, j))$, and for almost all $t \in$ $\left[t_{j+1}^{1}+t_{j}^{2}, t_{j+1}^{1}+t_{j+1}^{2}\right], \frac{d \bar{\phi}(t, j)}{d t}=\left(\frac{d \phi_{1}\left(\tau_{1}(t), j\right)}{d t}, \frac{d \phi_{2}\left(\tau_{2}(t), j\right)}{d t}\right)=$ $\left(0, \frac{d \phi_{2}\left(\tau_{2}, j\right)}{d \tau_{2}}\right) \in\left(0, F\left(\phi_{2}\left(\tau_{2}(t), j\right)\right)\right) \subset F_{j}(\bar{\phi}(t, j))$ and (iii) for any $(t, j) \in \operatorname{dom} \bar{\phi}$ such that $(t, j+1) \in$ $\operatorname{dom} \bar{\phi}, \bar{\phi}(t, j+1)=\left(\phi_{1}\left(\tau_{1}(t), j+1\right), \phi_{2}\left(\tau_{2}(t), j+1\right)\right) \in$ $\left(G\left(\phi_{1}\left(\tau_{1}(t), j\right)\right), G\left(\phi_{2}\left(\tau_{2}(t), j\right)\right)\right)=G_{\mathrm{j}}(\bar{\phi}(t, j))$. By (33), for each $(t, j) \in \operatorname{dom} \phi_{1}$, we can select $(\bar{t}, j) \in \operatorname{dom} \bar{\phi}$ such that $\tau_{1}(\bar{t})=t$. Selecting $t^{\prime}=\tau_{2}(\bar{t}),(12)$ follows from (31).

To prove (ii), we consider any solution $\bar{\phi}$ to (10) and introduce the jump time sequence $\left\{\bar{t}_{j}\right\}_{j \in \mathbb{N}_{0}}$ such that $\operatorname{dom} \bar{\phi}=$ $\bigcup_{j=0}^{\infty}\left[\bar{t}_{j}, \bar{t}_{j+1}\right] \times\{j\}$. Since $\bar{\phi}$ is a solution, $\frac{d \bar{\phi}}{d t} \in F_{\mathrm{j}}(\bar{\phi}(t, j))$ for almost all $t \in I^{j}:=\{t:(t, j) \in \operatorname{dom} \bar{\phi}\}$ and any $j \in \mathbb{N}_{0}$. Hence, by (10), and since $F_{\mathrm{j}}(q)$ is the convex hull of $\left(\bar{F}\left(q_{1}\right), 0\right)$ and $\left(0, \bar{F}\left(q_{2}\right)\right)$, there exists a function $\alpha_{1}:\left[0, \sup _{t} \operatorname{dom} \bar{\phi}\right] \rightarrow[0,1]$ such that

$$
\frac{d \bar{\phi}(t, j)}{d t} \in\left(\alpha_{1}(t) F\left(\bar{\phi}_{1}(t, j)\right),\left(1-\alpha_{1}(t)\right) F\left(\bar{\phi}_{2}(t, j)\right)\right)
$$

for almost all $t \in I^{j}=\{t:(t, j) \in \operatorname{dom} \bar{\phi}\}$ and all $j \in \mathbb{N}_{0}$. Introducing $\mu_{1}, \mu_{2}:\left[0, \sup _{t} \operatorname{dom} \bar{\phi}\right] \rightarrow \mathbb{R}_{>0}$ as $\mu_{1}(t)=\int_{0}^{t} \alpha_{1}(s) d s$ and $\mu_{2}(t)=t-\mu_{1}(t)$, from (35) we deduce $\frac{d \bar{\phi}_{i}(t, j)}{d t} \in F\left(\bar{\phi}_{i}(t, j)\right) \frac{d \mu_{i}}{d t}, i=1,2$ and, consequently,

$$
\frac{d \bar{\phi}_{i}(t, j)}{d \mu_{i}} \in F\left(\bar{\phi}_{i}(t, j)\right) \text {. }
$$

Further, the sets $\operatorname{dom} \phi_{i}=\left\{(t, j): t=\mu_{i}(\bar{t}),(\bar{t}, j) \in\right.$ $\operatorname{dom} \bar{\phi}\}, i=1,2$, are hybrid time domains as $\mu_{1}, \mu_{2}$ are continuous, non-decreasing functions. Introducing $\tau_{i}^{+}(\bar{t})=$ $\min \left\{t: t=\tau_{i}(\bar{t}),(\bar{t}, j) \in \operatorname{dom} \bar{\phi}\right\}, i=1,2$, we define

$$
\phi_{1}(t, j)=\bar{\phi}_{1}\left(\tau_{1}^{+}(t), j\right) \text { and } \phi_{2}(t, j)=\bar{\phi}_{2}\left(\tau_{2}^{+}(t), j\right) \text {. }
$$

We remark that if $\mu_{1}^{+}(\cdot)$ is discontinuous at $\tilde{t}$, such that $\bar{t}^{+}:=\lim _{t \searrow \tilde{t}} \mu^{+}(t)$ differs from $\bar{t}^{-}:=\lim _{t \nearrow \tilde{t}} \mu^{+}(t)$, then we observe that $\dot{\mu}(t)=\alpha(t)=0$ for $t \in\left[\bar{t}^{-}, \bar{t}^{+}\right]$. Hence, for every $j \in \mathbb{N}_{0}$, the function $t \mapsto \phi_{1}(t, j)=\bar{\phi}_{1}\left(\mu_{1}^{+}(t), j\right)$ is absolutely continuous and, similarly, we obtain that $t \mapsto$ $\phi_{2}(t, j)=\bar{\phi}_{2}\left(\mu_{2}^{+}(t), j\right)$ is absolutely continuous.

To conclude this proof, we observe that (37) defines two solutions to the hybrid system (1), since (i): $\phi_{1}(0,0), \phi_{2}(0,0) \in$ $C \cup D$ as $\bar{\phi}(0,0) \in C_{\mathrm{j}} \cup D_{\mathrm{j}}$, (ii): (36) holds and (iii): for all 
$(t, j) \in \operatorname{dom} \phi_{i}, i=1,2$, such that $(t, j+1) \in \operatorname{dom} \phi_{i}$, it holds that $\phi_{i}(t, j+1) \in G\left(\phi_{i}(t, j)\right)$ by the design of $G_{\mathrm{j}}$.

Proof of Lemma 4: To prove the only if-statement, we suppose, for the sake of contradiction, that there exists a maximal solution $\bar{\phi}$ to (10) such that $\sup _{j} \operatorname{dom} \bar{\phi}=J<\infty$

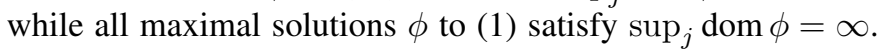
Let $\phi_{1}, \phi_{2}$ be maximal solutions to (1) as in item (ii) of Lemma 3. Introducing $T=\sup _{t} \operatorname{dom} \bar{\phi}$, we distinguish two cases.

If $T=\infty$, there has to exist a sequence $\left(T_{i}, J\right) \in \operatorname{dom} \bar{\phi}$ such that $\lim _{i \rightarrow \infty} T_{i}=T$. By applying item (ii) of Lemma 3, for each of these hybrid time instants (i.e. for every $i \in \mathbb{N}_{0}$ ), we find that there exists $\left(t_{i}, J\right) \in \operatorname{dom} \phi_{1},\left(t_{i}^{\prime}, J\right) \in \operatorname{dom} \phi_{2}$ such that $t_{i}+t_{i}^{\prime}=T_{i}$. Since $\lim _{i \rightarrow \infty} T_{i}=\infty$, we find either $\lim _{i \rightarrow \infty} t_{i}=\infty$, such that $\left(t_{i}, J\right) \in \operatorname{dom} \phi_{1}$ implies dom $\phi_{1}$ is bounded in the $j$-direction, or $\lim _{i \rightarrow \infty} t_{i}^{\prime}=\infty$ and dom $\phi_{2}$ is bounded in the $j$-direction. Hence, a contradiction is obtained with the assumption that $\sup _{j} \operatorname{dom} \phi_{1}=\sup _{j} \operatorname{dom} \phi_{2}=\infty$.

If $T \neq \infty$, we observe that the solutions $\phi_{1}, \phi_{2}$ to (1) satisfy $\sup _{j} \operatorname{dom} \phi=\infty$. Given $(T, J)$ and considering item (ii) of Lemma 3 , let $\bar{t}, \bar{t}^{\prime}$ be such that $\bar{\phi}(T, J)=\left(\phi_{1}(\bar{t}, J), \phi_{2}\left(\bar{t}^{\prime}, J\right)\right)$ and $T=\bar{t}+\bar{t}^{\prime}$. We define $T_{J}=\max \left\{t:(t, J) \in \operatorname{dom} \phi_{1}\right\}$ and $T_{J}^{\prime}=\max \left\{t:(t, J) \in \operatorname{dom} \phi_{2}\right\}$ and note that these maxima exist since $\sup _{j} \operatorname{dom} \phi_{1}=\sup _{j} \operatorname{dom} \phi_{2}=\infty$ by assumption. Observing $\bar{t} \leq T_{J}, \bar{t}^{\prime} \leq T_{J}^{\prime}$ and $T \leq T_{J}+T_{J}^{\prime}$, we define an extension to $\overline{\bar{\phi}}$ for hybrid times $(t, \bar{j}) \in \operatorname{dom} \bar{\phi}_{e}:=\operatorname{dom} \bar{\phi} \cup$ $\left[T, T_{J}+T_{J}^{\prime}\right] \times\{J\} \cup\left\{T_{J}+T_{J}^{\prime}\right\} \times\{J+1\}$ as

$$
\bar{\phi}_{e}(t, j)=\left\{\begin{array}{c}
\bar{\phi}(t, j), \\
\quad \text { for } j \leq J \text { and } t \leq T \\
\left(\phi_{1}((t-T)+\bar{t}, J), \phi_{2}\left(\bar{t}^{\prime}, J\right)\right), \\
\quad \text { for } j=J \text { and } T \leq t \leq \bar{t}^{\prime}+T_{J}, \\
\left(\phi_{2}\left(T_{J}, J\right), \phi_{2}\left(t-T_{J}, J\right)\right), \\
\quad \text { for } j=J \text { and } \bar{t}^{\prime}+T_{J} \leq t \leq T_{J}+T_{J}^{\prime}, \\
\left(\phi_{1}\left(T_{J}, J+1\right), \phi_{2}\left(T_{J}^{\prime}, J+1\right)\right), \\
\quad \text { for } j=J+1 \text { and } t=T_{J}+T_{J}^{\prime} .
\end{array}\right.
$$

As $\bar{\phi}_{e}$ is a solution to (10), $\bar{\phi}$ is not a maximal solution and a contradiction is attained. As in both cases a contradiction is found, the only if-statement is proved.

To prove the if-statement, suppose, for the sake of contradiction, that all maximal solutions $\bar{\phi}$ to (10) satisfy $\sup _{j} \operatorname{dom} \bar{\phi}=\infty$ and there exist some maximal solution $\phi_{1}$ to (1) for which $\sup _{j} \operatorname{dom} \phi_{1}=J \neq \infty$.

Define the hybrid time domain $\operatorname{dom} \bar{\phi}=\left\{(t, j) \in \mathbb{R}_{>0} \times\right.$ $\left.\mathbb{N}_{0}:\left(\frac{t}{2}, j\right) \in \operatorname{dom} \phi_{1}\right\}$ and for $(t, j) \in \operatorname{dom} \bar{\phi}$, we define the hybrid arc

$$
\bar{\phi}(t, j)=\left(\phi_{1}\left(\frac{t}{2}, j\right), \phi_{1}\left(\frac{t}{2}, j\right)\right)
$$

and observe that $\bar{\phi}$ is a solution to (10). By assumption, we can extend this solution to obtain $\bar{\phi}_{e}$ with $\sup _{j} \operatorname{dom} \bar{\phi}_{e}=\infty$. From (10), we find that there exists an integrable function $\alpha_{1}:\left[0, \sup _{t} \operatorname{dom} \bar{\phi}_{e}\right] \rightarrow[0,1]$ such that $\frac{d \bar{\phi}_{e}(t, j)}{d t}=$ $\left(\alpha_{1}(t) \bar{F}\left(\bar{\phi}_{1 e}(t, j)\right),\left(1-\alpha_{1}(t)\right) \bar{F}\left(\bar{\phi}_{1 e}(t, j)\right)\right)$ for some $j$ such that $(t, j) \in \operatorname{dom} \bar{\phi}_{e}$ and almost all $t \in\left[0, \sup _{t} \operatorname{dom} \bar{\phi}_{e}\right]$. Introducing $\tau_{1}(t)=\int_{0}^{t} \alpha_{1}(s) d s$ and the hybrid time domain $\operatorname{dom} \phi_{1 e}=\left\{(t, j) \in \mathbb{R}_{\geq 0} \times \mathbb{N}_{0}: t=\tau_{1}(\bar{t}),(\bar{t}, j) \in \operatorname{dom} \phi_{1}\right\}$ we can define the function $\phi_{1 e}(t, j)=\bar{\phi}_{1 e}\left(\tau^{+}(t), j\right)$, with $\tau_{1}^{+}(s)=\min \left\{t: \tau_{1}(t)=s\right\}$. It can be observed that $\phi_{1 e}(t, j)$ is a solution to (1). Since $\phi_{1 e}(t, j)=\phi_{1}(t, j)$ for $(t, j) \in$ $\operatorname{dom} \phi_{1} \subset \operatorname{dom} \phi_{1 e}$ and $\sup _{j} \operatorname{dom} \phi_{1 e}=\sup _{j} \operatorname{dom} \bar{\phi}=\infty$, we conclude that $\phi_{1 e}$ is an extension of $\phi_{1}$ and $\phi_{1}$ is not maximal, yielding a contradiction. This contradiction proves the if-statement.

Proof of Theorem 3: The proof of this Theorem follows the same steps as the proof of Theorem 1 by relying on Lemmas 3 and 4 and is omitted for the sake of brevity.

Proof of Proposition 4: The proof of this proposition is omitted and follows the same steps as Proposition 2.

Proof of Proposition 5: Suppose system (1) is $\delta$-UpIS with $\delta \in$ $\mathcal{D}$. Item (i) of Definition 3 immediately implies the satisfaction of items (i) in Definitions 4 and 6.

Let $\varepsilon, r>0$, and take $\Theta$ as in item (ii) of Definition 3 . Define $T=\Theta$, and let $\left(\phi_{1}, \phi_{2}\right)$ be a pair of maximal solutions to system (1). For all $(t, j) \in \operatorname{dom} \phi_{1}$ with $t \geq T$, it holds that $t+j \geq \Theta$ by definition of $T$. We then know from item (ii) of Definition 3 that there exists $\left(t^{\prime}, j\right) \in \operatorname{dom} \phi_{2}$ with $\left|t-t^{\prime}\right|<\varepsilon$ such that $\delta\left(\phi_{1}(t, j), \phi_{2}\left(t^{\prime}, j\right)\right)<\varepsilon$. Hence item (ii) of Definition 4 holds. The same reasoning is used to prove that item (ii) of Definition 6 is verified. This ensures item (i) of Proposition 5 holds.

From the above, item (ii) of Proposition 5 is also verified. Indeed, if system (1) is $\delta$-UIS, it is necessarily $\delta$-UpIS and thus $\delta$-FUpIS and $\delta$-JUpIS in view of item (i) of Proposition 5. In addition, since any maximal solution $\phi$ to (1) is complete, for all maximal solutions $\phi$ either $\sup _{t} \operatorname{dom} \phi=\infty$ or $\sup _{j} \operatorname{dom} \phi=\infty$ or both. Note that we cannot have maximal solutions $\phi_{1}, \phi_{2}$ with $\sup _{t} \operatorname{dom} \phi_{1}=\infty$ and $\sup _{t} \operatorname{dom} \phi_{2}<$ $\infty$, and $\sup _{j} \operatorname{dom} \phi_{1}<\infty$ and $\sup _{j} \operatorname{dom} \phi_{2}=\infty$ as the system would not be $\delta$-FUpIS and $\delta$-JUpIS respectively, as explained after Definitions 4 and 6. Hence, the system is either $\delta$-FUIS or $\delta$-JUIS.

Let us now prove item (iii) of Proposition 5. We construct a system which is both $\delta$-JUpIS and $\delta$-FUpIS with a given $\delta$ but which is not $\delta$-UpIS. Let

$$
\dot{\tau}=1, \text { for } \tau \in[0,1] \quad \tau^{+}=0, \text { for } \tau=1 .
$$

Let $\delta\left(\tau_{1}, \tau_{2}\right)=0$ for any $\tau_{1}, \tau_{2} \in \mathbb{R}$, and $\left(\phi_{1}, \phi_{2}\right)$ be a pair of maximal solutions to (39). For any $(t, j) \in \operatorname{dom} \phi_{1}$, there exists $\left(t, j^{\prime}\right) \in \operatorname{dom} \phi_{2}$ (since any maximal solution to (39) is $t$-complete) and $\delta\left(\phi_{1}(t, j), \phi_{2}\left(t, j^{\prime}\right)\right)=0$. We deduce that system (39) is $\delta$-FUIS in view of the particular form of $\delta$. We similarly derive that system (39) is $\delta$-JUIS. However, this system is not $\delta$-UpIS. Indeed, consider item (i) of Definition 3 and let $\varepsilon=\frac{1}{4}$ and $\phi_{1}$ and $\phi_{2}$ be two maximal solutions with $\phi_{1}(0,0)=0$ and $\phi_{2}(0,0)=1$. We note that $\delta\left(\phi_{1}(0,0), \phi_{2}(0,0)\right)=0<r$ for any $r>0$. For $\left(\frac{1}{2}, 0\right) \in \operatorname{dom} \phi_{1}$, there does not exist $\left(t^{\prime}, 0\right) \in \operatorname{dom} \phi_{2}$ such that $\left|\frac{1}{2}-t^{\prime}\right|<\varepsilon$ as required in item (i) of Definition 3. Indeed, $\operatorname{dom} \phi_{2}=(\{0\} \times\{0\}) \times([0,1] \times\{1\}) \times \ldots$, so the only time $t^{\prime}$ such that $\left(t^{\prime}, 0\right) \in \operatorname{dom} \phi_{2}$ is $t^{\prime}=0$ but $\left|\frac{1}{2}-t^{\prime}\right|=\frac{1}{2}>\frac{1}{4}=\varepsilon$. An illustration of the hybrid time domains of $\phi_{1}$ and $\phi_{2}$ is given in Figure 1. Consequently, system (39) is both $\delta$-FU(p)IS and $\delta$-JU(p)IS, but not $\delta$-(p)UIS.

Proof of Proposition 6: Consider hybrid system (1) with the data given in Proposition 6. For any pair of maximal solutions $\left(\phi_{1}, \phi_{2}\right), \operatorname{dom} \phi_{1}=\operatorname{dom} \phi_{2}=\mathbb{R}_{\geq 0} \times\{0\}$. For any $(t, 0) \in$ 


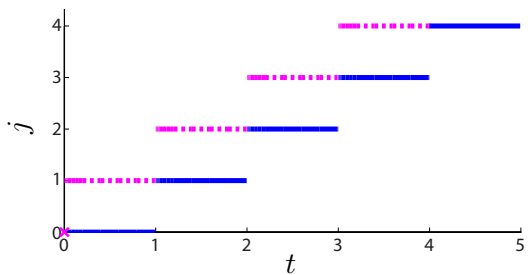

Figure 1. Hybrid time domains of the maximal solutions to (39) initialised at $\phi_{1}(0,0)=0$ (blue solid lines) and at $\phi_{2}(0,0)=1$ (magenta dashed lines)

$\operatorname{dom} \phi_{1}=\operatorname{dom} \phi_{2}$

$$
\delta\left(\phi_{1}(t, 0), \phi_{2}(t, 0)\right) \leq \beta\left(\delta\left(\phi_{1}(0,0), \phi_{2}(0,0)\right), t\right)
$$

in view of (16), from which we deduce that items (i) and (ii) of Definition 3 hold (by respectively taking $s$ such that $\beta(s, 0)<\varepsilon$ and $\left(t^{\prime}, j\right)=(t, j)$, and $\Theta$ such that $\beta(r, \Theta)<$ $\varepsilon)$. Therefore, the hybrid system is $\delta$-UpIS and thus $\delta$-FUpIS according to Proposition 5. The system is $\delta$-UIS and $\delta$-FUIS since the solutions are $t$-complete.

Proof of Proposition 7: Consider hybrid system (1) with the data given in Proposition 7. For any pair of maximal solutions $\left(\phi_{1}, \phi_{2}\right), \operatorname{dom} \phi_{1}=\operatorname{dom} \phi_{2}=\{0\} \times \mathbb{N}_{0}$. For any $(0, j) \in \operatorname{dom} \phi_{1}=\operatorname{dom} \phi_{2}, \delta\left(\phi_{1}(0, j), \phi_{2}(0, j)\right) \leq$ $\beta\left(\delta\left(\phi_{1}(0,0), \phi_{2}(0,0)\right), j\right)$ in view of $(17)$, from which we deduce that items (i) and (ii) of Definition 3 hold (by respectively taking $s$ such that $\beta(s, 0)<\varepsilon$ and $\left(t^{\prime}, j\right)=(0, j)$, and $\Theta$ such that $\beta(r, \Theta)<\varepsilon)$. Therefore, the hybrid system is $\delta$-UpIS and thus $\delta$-JUpIS according to Proposition 5. The system is $\delta$-UIS and $\delta$-JUIS since the solutions are $j$-complete.

Proof of Theorem 2: Let $\delta$ be the Euclidean distance and let $\epsilon>0$ be given. Applying [37, Theorem 4], we find that there exists $s>0$ such that for any pair of maximal solutions $\left(\phi_{1}, \phi_{2}\right)$ to $(1)$, the conditions $\left\|\phi_{1}(0,0)-\phi_{2}(0,0)\right\|<s$ and

$$
\begin{aligned}
& \forall(t, j) \in \operatorname{dom} \phi_{1}, \exists\left(t, j^{\prime}\right) \in \operatorname{dom} \phi_{2} \text { such that } \\
& \rho_{\mathcal{A}}\left(\phi_{1}(t, j), \phi_{2}\left(t, j^{\prime}\right)\right)<s
\end{aligned}
$$

imply that, for all $(t, j) \in \operatorname{dom} \phi_{1}$, there exists $\left(t^{\prime}, j^{\prime \prime}\right) \in$ $\operatorname{dom} \phi_{2}$ with $\left|t-t^{\prime}\right| \leq \epsilon$ and $\left\|\phi_{1}(t, j)-\phi_{2}\left(t^{\prime}, j^{\prime \prime}\right)\right\| \leq \epsilon$, as required in item (i) of Definition 4. Since system (1) is $\rho_{\mathcal{A}}$-tFUIS, in view of item (i) of Definition 4 , we can find a scalar $\bar{s}>0$ such that $\left\|\phi_{1}(0,0)-\phi_{2}(0,0)\right\|<\bar{s}$ (trivially implying $\left.\rho_{\mathcal{A}}\left(\phi_{1}(0,0), \phi_{2}(0,0)\right)<\bar{s}\right)$ implies that $(41)$ is satisfied. Consequently, if $\left\|\phi_{1}(0,0)-\phi_{2}(0,0)\right\| \leq \min (s, \bar{s})$, then item (i) of Definition 4 holds.

To prove item (ii) of Definition 4, we take $\epsilon, r>0$ and select $s$ as above. With [37, Lemma 5], we can show that there exists some positive scalar $\tilde{s} \leq s$ such that for every pair of maximal solutions $\tilde{\phi}_{1}, \tilde{\phi}_{2}$ with $\rho_{\mathcal{A}}\left(\tilde{\phi}_{1}(t, j), \tilde{\phi}_{2}\left(t, j^{\prime}\right)\right)<\tilde{s}$ for all $(t, j) \in \operatorname{dom} \tilde{\phi}_{1}$ and some $\left(t, j^{\prime}\right) \in \operatorname{dom} \tilde{\phi}_{2}$, there exists $\tilde{t} \in[0,1]$ and $(\tilde{t}, \tilde{j}) \in \operatorname{dom} \tilde{\phi}_{1},\left(\tilde{t}, \tilde{j}^{\prime}\right) \in \operatorname{dom} \tilde{\phi}_{2}$ such that $\left\|\tilde{\phi}(\tilde{t}, \tilde{j})-\tilde{\phi}_{2}\left(\tilde{t}, \tilde{j}^{\prime}\right)\right\| \leq s$.

Now, select $\bar{T}$ such that $\left\|\phi_{1}(0,0)-\phi_{2}(0,0)\right\| \leq r$, (which trivially means $\rho_{\mathcal{A}}\left(\phi_{1}(0,0), \phi_{2}(0,0)\right) \leq r$ ) implies $\rho_{\mathcal{A}}\left(\phi_{1}(t, j), \phi_{2}\left(t, j^{\prime}\right)\right) \leq \tilde{s}$, with $(t, j) \in \operatorname{dom} \phi_{1}$ and $\left(t, j^{\prime}\right) \in$ $\operatorname{dom} \phi_{1}$ and $t \geq \bar{T}$ (such $\bar{T}$ exists by $\rho_{\mathcal{A}}$-tFUIS). With the selection of $\tilde{s}$ as above and observing that solutions $\phi_{1}(t, j), \phi_{2}\left(t, j^{\prime}\right)$ from time $(T, j)$ and $\left(T, j^{\prime}\right)$ can be extended by reparameterising solutions $\tilde{\phi}_{1}, \tilde{\phi}_{2}$ as above, we find that there exists some $\tilde{T} \leq \bar{T}+1,(\tilde{T}, \tilde{J}) \in \operatorname{dom} \phi_{1}$ and $\left(\tilde{T}, \tilde{J}^{\prime}\right) \in \operatorname{dom} \phi_{2}$ such that $\left\|\phi_{1}(\tilde{T}, \tilde{J})-\phi_{2}\left(\tilde{T}, \tilde{J}^{\prime}\right)\right\| \leq s$ and $\rho_{\mathcal{A}}\left(\phi_{1}(t, j), \phi_{2}\left(t, j_{\sim}^{\prime}\right)\right) \leq s$ hold for all $(t, j) \in \operatorname{dom} \phi_{1},\left(t, j^{\prime}\right) \in$ dom $\phi_{2}$ with $t \geq \tilde{T}$. Hence, the design of $s$ as above implies that item (ii) of Definition 4 holds for $T=\bar{T}+1$ and $\delta$ the Euclidean distance, concluding the proof.

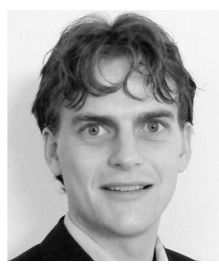

Benjamin Biemond received the M.Sc. and Ph.D. degrees in Mechanical Engineering from the Eindhoven University of Technology, Eindhoven, the Netherlands in 2009 and 2013, respectively. From 2013 to 2016, he was affiliated with the Department of Computer Science at KU Leuven in Belgium as postdoctoral researcher. Since 2016, he is with the Department of Optomechatronics, Netherlands Organization for Applied Scientific Research, TNO, Delft, the Netherlands. His research interests include dynamics, stability, and control of nonlinear systems, including nonsmooth and hybrid systems as well as time-delay systems. He was a recipient of a personal Pegasus Marie Curie grant awarded by the Research Foundation-Flanders (FWO).

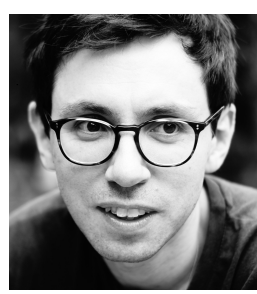

Romain Postoyan received the M.Sc. degree in Electrical and Control Engineering from ENSEEIHT (France) in 2005. He obtained the M.Sc. by Research in Control Theory \& Application from Coventry University (United Kingdom) in 2006 and the Ph.D. in Control Theory from Université ParisSud (France) in 2009. In 2010, he was a research assistant at the University of Melbourne (Australia). Since 2011, he is a CNRS researcher at the Centre de Recherche en Automatique de Nancy (France).

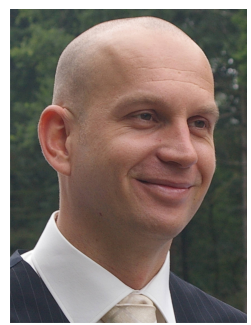

Maurice Heemels (F'15) received the M.Sc. degree in mathematics and the Ph.D. degree in control theory (both summa cum laude) from the Eindhoven University of Technology (TU/e), Eindhoven in 1995 and 1999, respectively. From 2000 to 2004, he was with the Electrical Engineering Department, TU/e and from 2004 to 2006 with the Embedded Systems Institute (ESI). Since 2006, he has been with the Department of Mechanical Engineering, TU/e, where he is currently a Full Professor with the Control Systems Technology Group. Dr. Heemels was a recipient of a personal VICI grant from the Netherlands Organization for Scientific Research and the Dutch Technology Foundation.

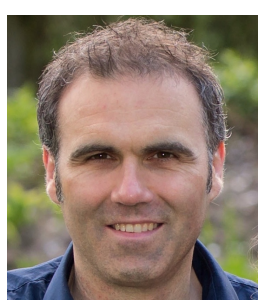

Nathan van de Wouw obtained his M.Sc.-degree (with honors) and Ph.D.-degree in Mechanical Engineering from the Eindhoven University of Technology, the Netherlands, in 1994 and 1999, respectively. He currently holds a full professor position at the Mechanical Engineering Department of the Eindhoven University of Technology, the Netherlands. Nathan van de Wouw also holds an adjunct full professor position at the University of Minnesota, U.S.A. and a (part-time) full professor position at the Delft University of Technology, the Netherlands. In 2015, he received the IEEE Control Systems Technology Award. 